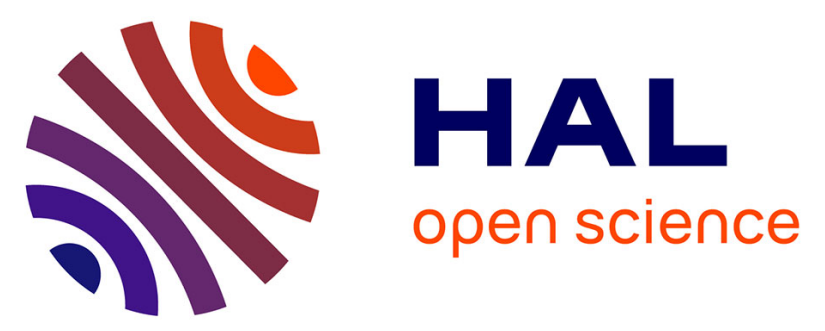

\title{
Barotropic, baroclinic, and inertial instabilities of the easterly Gaussian jet on the equatorial $\beta$-plane in rotating shallow water model
}

\author{
Bruno Ribstein, Vladimir Zeitlin, Ann-Sophie Tissier
}

\section{To cite this version:}

Bruno Ribstein, Vladimir Zeitlin, Ann-Sophie Tissier. Barotropic, baroclinic, and inertial instabilities of the easterly Gaussian jet on the equatorial $\beta$-plane in rotating shallow water model. Physics of Fluids, 2014, 26 (5), pp.56605. 10.1063/1.4875030 . hal-01083037

\section{HAL Id: hal-01083037}

https://hal-polytechnique.archives-ouvertes.fr/hal-01083037

Submitted on 14 Nov 2014

HAL is a multi-disciplinary open access archive for the deposit and dissemination of scientific research documents, whether they are published or not. The documents may come from teaching and research institutions in France or abroad, or from public or private research centers.
L'archive ouverte pluridisciplinaire HAL, est destinée au dépôt et à la diffusion de documents scientifiques de niveau recherche, publiés ou non, émanant des établissements d'enseignement et de recherche français ou étrangers, des laboratoires publics ou privés. 


\section{AIP $\left.\right|_{\text {Physics of }}$ \\ Fluids}

\section{Barotropic, baroclinic, and inertial instabilities of the easterly Gaussian jet on the equatorial $\beta$-plane in rotating shallow water model}

Bruno Ribstein, Vladimir Zeitlin, and Ann-Sophie Tissier

Citation: Physics of Fluids (1994-present) 26, 056605 (2014); doi: 10.1063/1.4875030

View online: http://dx.doi.org/10.1063/1.4875030

View Table of Contents: http://scitation.aip.org/content/aip/journal/pof2/26/5?ver=pdfcov

Published by the AIP Publishing

\section{Articles you may be interested in}

Decaying vortex and wave turbulence in rotating shallow water model, as follows from high-resolution direct numerical simulations

Phys. Fluids 24, 115106 (2012); 10.1063/1.4767723

Inertial, barotropic, and baroclinic instabilities of the Bickley jet in two-layer rotating shallow water model

Phys. Fluids 23, 126601 (2011); 10.1063/1.3661995

Frequency selection in globally unstable round jets

Phys. Fluids 19, 054108 (2007); 10.1063/1.2732247

The baroclinic secondary instability of the two-dimensional shear layer

Phys. Fluids 12, 2489 (2000); 10.1063/1.1289503

Sideband instabilities of mixed barotropic/baroclinic waves growing on a midlatitude zonal jet

Phys. Fluids 9, 615 (1997); 10.1063/1.869221

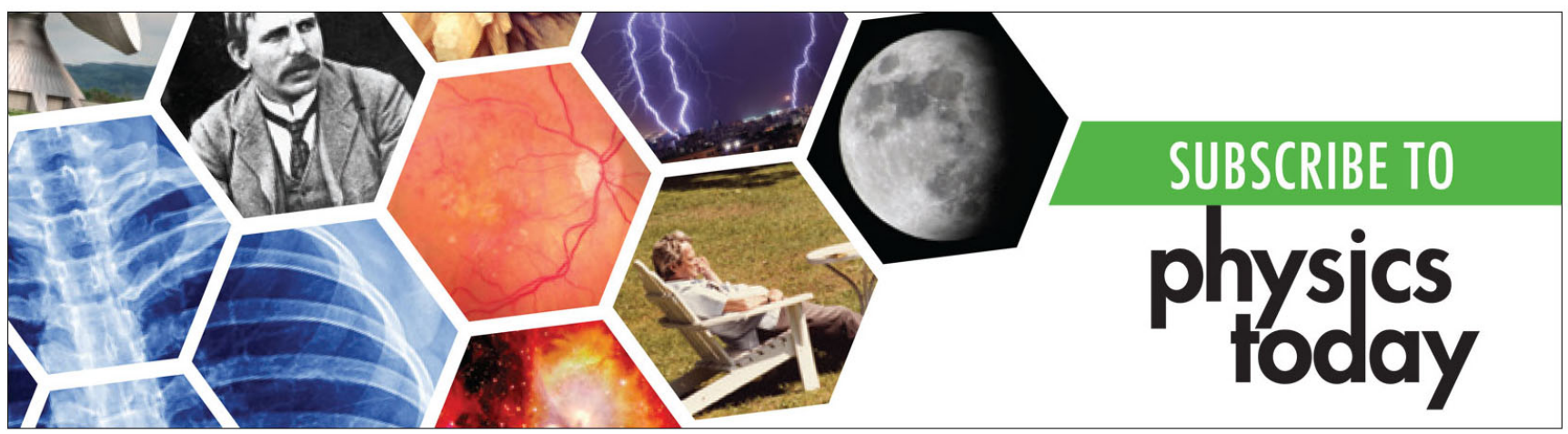




\title{
Barotropic, baroclinic, and inertial instabilities of the easterly Gaussian jet on the equatorial $\beta$-plane in rotating shallow water model
}

\author{
Bruno Ribstein, ${ }^{1}$ Vladimir Zeitlin, ${ }^{2, a)}$ and Ann-Sophie Tissier ${ }^{3}$ \\ ${ }^{1}$ Institut für Atmosphäre und Umwelt, Goethe-Universität, Altenhöferallee 1, \\ 60438 Frankfurt/Main, Germany \\ ${ }^{2}$ Institut Universitaire de France, and LMD, Univ. P. and M. Curie, BP 99, 5, \\ pl. Jussieu, 75005 Paris, France \\ ${ }^{3}$ LMD, Univ. P. et M. Curie, and ENS, 24 rue Lhomond, 75005 Paris, France
}

(Received 25 July 2013; accepted 17 April 2014; published online 20 May 2014)

\begin{abstract}
A detailed linear stability analysis of an easterly barotropic Gaussian jet centered at the equator is performed in the long-wave sector in the framework of one- and twolayer shallow-water models on the equatorial $\beta$-plane. It is shown that the dominant instability of the jet is due to phase-locking and resonance between Yanai waves, although the standard barotropic and baroclinic instabilities due to the resonance between Rossby waves are also present. In the one-layer case, this dominant instability has non-zero growth rate at zero wavenumber for high enough Rossby and low enough Burger numbers, thus reproducing the classical symmetric inertial instability. Yet its asymmetric counterpart has the highest growth rate. In the two-layer case, the dominant instability may be barotropic or baroclinic, the latter being stronger, with the maximum of the growth rate shifting towards smaller downstream wavenumbers as Rossby number increases at fixed Burger number, and given thickness and density ratios. At large enough Rossby numbers this instability has a non-zero growth rate limit at zero wavenumber, giving the baroclinic symmetric inertial instability. Again, the maximal growth rate is achieved at small but non-zero wavenumbers, corresponding to the asymmetric inertial instability. At high enough Rossby number and low enough Burger number not only the baroclinic, but also the barotropic symmetric instability appears, as well as higher meridional modes of the baroclinic symmetric instability. Still, all of them are dominated by their asymmetric counterparts. Direct numerical simulations of the saturation of the leading instabilities are performed, showing that the barotropic species of the instability saturates by forming a double vortex street subject to nonlinear oscillations, while the baroclinic, the most vigorous one, saturates by producing strong vertical shears and related dissipation and mixing. (c) 2014 AIP Publishing LLC. [http://dx.doi.org/10.1063/1.4875030]
\end{abstract}

\section{INTRODUCTION}

It is well known that the atmosphere and the ocean in the equatorial region exhibit characteristic zonal jet structures. Instabilities of equatorial jets play an important role in the dynamics of this region, see, e.g., Ref. 1 for the ocean, and, e.g., Ref. 2 for the troposphere. They are also important in the context of general circulation of the Earth and other planets, see, e.g., Ref. 3. The equatorial region, due to the vanishing Coriolis parameter, favors the inertial instability, e.g., Refs. 4 and 5. Yet, systematic studies of the instabilities of jets at the equator are rather scarce, as to our knowledge. In particular, the interplay between ageostrophic baroclinic and barotropic instabilities, on the one hand, and the inertial instability, on the other hand, which was a subject of active investigation on

a) zeitlin@1md.ens.fr 
the mid-latitude tangent plane recently ${ }^{6-8}$ was not sufficently studied at the equator. In the present paper, we undertake an investigation of the instabilities of a zonal easterly jet centered at the equator, a configuration which is relevant in the atmospheric context, e.g., Ref. 9. We chose the simplest, yet frequently used for modeling of this region, e.g., Refs. 1, 10, and 11, rotating shallow model on the equatorial $\beta$-plane in its barotropic (one-layer) and baroclinic (two-layer) versions, and perform a detailed linear stability analysis of the barotropic Gaussian jet with special attention to the long-wave sector, which is synoptically most important. Following the lines of Ref. 6, where such approach was realized on the mid-latitude tangent plane ( $f$-plane), we identify and classify the instabilities as resonances of the linear wave-modes propagating on the background of the jet in both versions of the model by using the pseudo-spectral collocation technique. The rich spectrum of equatorial waves, e.g., Ref. 12, leads to a large variety of possible resonances, especially in the baroclinic case, thus complicating substantially the task, as compared to the $f$-plane case. We then study nonlinear saturation of the instabilities with the help of the new-generation finite-volume numerical scheme. ${ }^{13}$

In what concerns the linear stability analysis in shallow water, the approach we adopted was initiated in the pioneering paper ${ }^{14}$ on the stability of double fronts with zero potential vorticity, i.e., of terminating (in- or outcropping) layers of shallow water on the equatorial $\beta$-plane, and extended to fronts with non-zero potential vorticity in Ref. 15. (However, we should immediately stress that we do not consider in- or outcropping layers below.) This approach was recently applied, using the one-layer rotating shallow water model, to the instabilities of shear flows on the equatorial $\beta$-plane ${ }^{16}$ and on the sphere ${ }^{17}$ in the context of wind distribution in planetary atmospheres. The paper ${ }^{16}$ is closest in spirit to what follows, although the precise nature of the instabilities of our flow configuration appears to be different. While revising the present paper we learned about the work ${ }^{18}$ on purely symmetric equatorial inertial instability and its nonlinear saturation.

The paper is organized as follows. In Sec. II, we present the model and describe the background flow. In Secs. III and IV, respectively, we present the results of the linear stability analysis in the barotropic and the baroclinic versions of the model. Section V contains the results of direct numerical simulations of the saturation of typical instabilities. Summary and discussion are given in Sec. VI.

\section{THE MODEL AND THE BACKGROUND FLOW}

\section{A. One-layer configuration}

The equations of the one-layer rotating shallow water model (RSW in what follows) on the equatorial $\beta$-plane read

$$
\begin{aligned}
D_{t} \vec{v}+\beta y \overrightarrow{e_{z}} \times \vec{v}+g \vec{\nabla} h & =0, \\
\partial_{t} h+\partial_{x}(h u)+\partial_{y}(h v) & =0,
\end{aligned}
$$

where $\overrightarrow{e_{z}}$ is the unit vertical vector, $\beta y$ is the Coriolis parameter on the equatorial $\beta$-plane, $\vec{v}=(u, v)$ is the horizontal fluid velocity in the layer, $h$ is the layer depth, and $D_{t}=\partial_{t}+u \partial_{x}+v \partial_{y}$ is the Lagrangian (advective) derivative. It is worth recalling that the RSW model, both in one- and multilayer versions, follows from the vertical averaging of the three-dimensional hydrostatic primitive equations between a pair of material surfaces, e.g., Ref. 19.

The energy of the system, modulo a constant

$$
E=E_{k}+E_{p}=\int d x d y\left(\frac{h}{2} \vec{v}^{2}+g \frac{h^{2}}{2}\right)
$$

is conserved in the absence of dissipation. An important quantity in what follows is potential vorticity (PV) $q$, which is a Lagrangian invariant in the absence of dissipation

$$
q=\frac{\zeta+\beta y}{h}, \quad D_{t} q=0 .
$$

Here $\zeta=\partial_{x} v-\partial_{y} u$ is the relative vorticity and $\zeta+\beta y$ is the absolute vorticity. 
The background jet is an exact solution of (2.1),

$$
\begin{aligned}
& H(y)=H_{0}-\Delta H e^{-(y / L)^{2}}, \\
& U(y)=-2 U_{0} e^{-(y / L)^{2}}, \\
& V(y)=0,
\end{aligned}
$$

where $\Delta H \leq H_{0}$ and $U_{0}=\frac{g \Delta H}{\beta L^{2}}$. Here and below capitals $U, V, H$ are used for stationary solutions.

The problem can be non-dimensionalized by using the width of the jet $L$ as the horizontal length scale, $H_{0}$ as the vertical scale, (half of) the maximal jet velocity, $U_{0}$ as the velocity scale, and $\frac{L}{U_{0}}$ as the time scale. The dimensionless parameters are then the Rossby number $R o=\frac{U_{0}}{\beta L^{2}}$ and the Burger number $B u=\frac{g H_{0}}{\left(\beta L^{2}\right)^{2}}$. We also introduce the non-dimensional deviation of the free surface $\lambda=\frac{\Delta H}{H_{0}}=\frac{R o}{B u} \leq 1$ (this restriction excludes incropping flows). In what follows we work with non-dimensional equations, with $R o$ and $B u$ as main parameters. It should be emphasized that a combination of these parameters, in our context $E=\frac{R o^{4}}{B u}$, was used in Ref. 16 in the study of shear flows. We, however, find more convenient to work with the pair $R o-B u$ as each of them influences the instability of the jet in its own way, see below and Appendix A.

We should recall that in the context of the Earth atmosphere $\beta=2 \Omega / a_{0}$, where $\Omega=2 \pi /(24 \times$ $60 \times 60) \mathrm{s}^{-1}$ is the angular velocity of the Earth rotation, $a_{0}=6371 \mathrm{~km}$ is the radius of the Earth, and $g=9.81 \mathrm{~ms}^{-2}$. The depth of the tropical tropopause is about $20 \mathrm{~km}$, which may be taken as the "face value" of $H_{0}$ in the model. Yet, the shallow-water interpretations of the data on equatorial waves use the so-called equivalent depth, cf. Ref. 12, which may be of the order of tens of meters in the troposphere ${ }^{20}$ or several kilometers in the stratosphere ${ }^{21}$ for the observed waves. Scaling for equatorial motions is not unique, ${ }^{22}$ and it is not our goal here to adapt the model to a concrete observed phenomenon which, in any case, will fall into a regime with some given values of nondimensional parameters. That is why below all the calculations are made, and all results are presented, in non-dimensional terms.

The jet configurations in the geostrophic $(R o \rightarrow 0)$ and ageostrophic $(R o=\mathcal{O}(1))$ cases are presented in Fig. 1. Note the North-South $(y \rightarrow-y)$ symmetry of the background jet velocity and normalized vorticity. We also remark that the normalized absolute vorticity is not sign-definite in the ageostrophic configuration. We, thus, expect the appearance of inertial instability at $R o \geq 1 / 4$, at the central part of the jet

$$
f \times \zeta \leq 0 \Longleftrightarrow R o \geq 1 / 4
$$
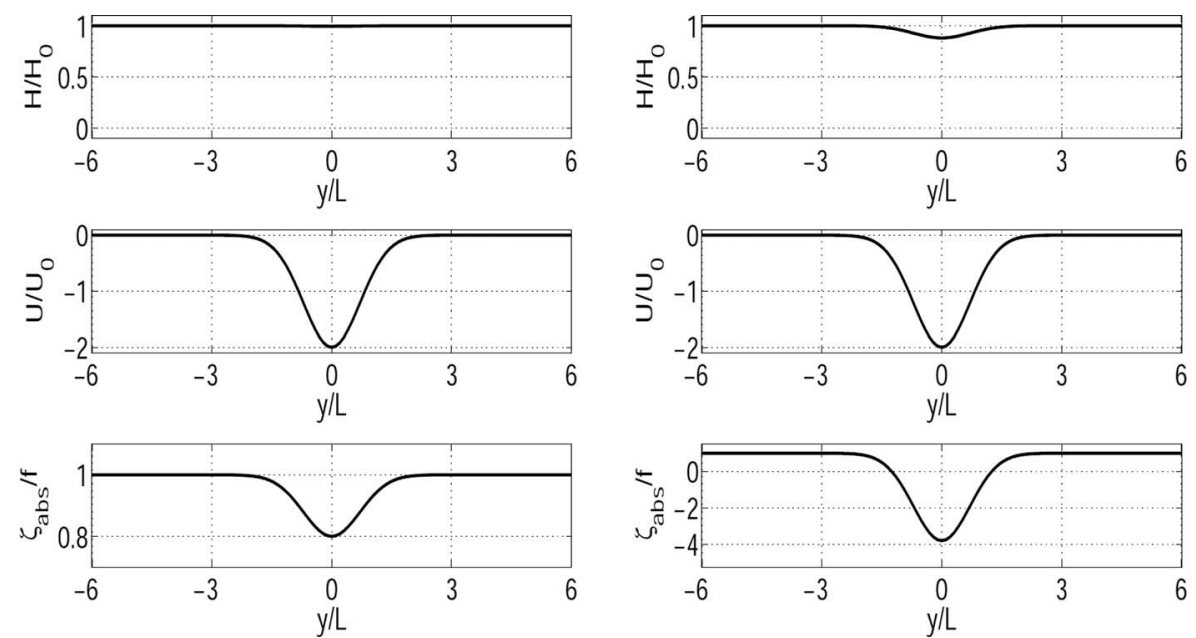

FIG. 1. Background flow with $B u=10$ at $R o=0.05$ (left column) and $R o=1.2$ (right column). (Upper panel) thickness; left (right) column: $\lambda=\frac{\Delta H}{H}=\frac{R o}{B u}=5 \times 10^{-3}(0.12)$; (Middle panel) zonal velocity profile; (Lower panel) profile of the absolute vorticity $\beta y+\zeta$ normalized by the planetary vorticity $\beta y$. 
cf., e.g., Ref. 23. Moreover, it is easy to check that the Ripa's general stability conditions, see Ref. 24 and Appendix B, are satisfied for $R o \leq 1 / 4$, but not for $R o>1 / 4$.

\section{B. Two-layer configuration}

The equations of the two-layer RSW on the equatorial $\beta$-plane can be written in the following form:

$$
\begin{aligned}
D_{i t} \overrightarrow{v_{i}}+\beta y \overrightarrow{e_{z}} \times \overrightarrow{v_{i}}+g \vec{\nabla}\left(\alpha_{i} h_{1}+h_{2}\right)=0, \\
\partial_{t} h_{i}+\partial_{x}\left(h_{i} u_{i}\right)+\partial_{y}\left(h_{i} v_{i}\right)=0,
\end{aligned}
$$

where the index $i=1,2$ refers to the upper and the lower layers, respectively, $D_{i t}=\partial_{t}+u_{i} \partial_{x}+v_{i} \partial_{y}$ are advective derivatives in the layers, $\alpha_{1} \equiv 1, \alpha_{2}=\frac{\rho_{1}}{\rho_{2}} \equiv \rho<1$, and $\rho_{i}, \overrightarrow{v_{i}}, h_{i}$ are the densities, the velocities and the thicknesses of the layers. The last terms in the momentum equations in (2.6) represent the pressure in the respective layers.

The energy of the system is a sum of potential and kinetic energies of the layers, $E=E_{1}+$ $E_{2}$, and is conserved in the absence of dissipation. The energies of the layers are defined, modulo a constant, as

$$
\left\{\begin{array}{l}
E_{1}=\int d x d y \rho_{1}\left[h_{1} \frac{\vec{v}_{1}^{2}}{2}+g h_{1} h_{2}+g \frac{h_{1}^{2}}{2}\right] \\
E_{2}=\int d x d y \rho_{2}\left[h_{2} \frac{\vec{v}_{2}^{2}}{2}+g \frac{h_{2}^{2}}{2}\right] .
\end{array}\right.
$$

The potential vorticities $q_{i}$ are Lagrangian invariants layerwise, in the absence of dissipation

$$
q_{i}=\frac{\zeta_{i}+\beta y}{h_{i}}, \quad D_{i t} q_{i}=0
$$

where no summation over repeated index is assumed.

We take a barotropic jet, which is an exact solution of (2.6), as the background flow

$$
\begin{aligned}
& H_{1}(y)=H_{10}, \\
& H_{2}(y)=H_{20}-\Delta H e^{-(y / L)^{2}}, \\
& U_{1}(y)=-2 U_{0} e^{-(y / L)^{2}}, \\
& U_{2}(y)=-2 U_{0} e^{-(y / L)^{2}}, \\
& V_{1}(y)=V_{2}(y)=0,
\end{aligned}
$$

where $\Delta H \leq H_{20}$ and $U_{0}=\frac{g \Delta H}{\beta L^{2}}$.

The problem is again non-dimensionalized using the horizontal length scale $L$, the full thickness $H_{0}=H_{10}+H_{20}$ as the vertical scale, the velocity scale $U_{0}$, and the time scale $\frac{L}{U_{0}}$. The dimensionless parameters are the Rossby number, the Burger number, and the aspect ratios $d_{i}=\frac{H_{i 0}}{H_{0}}$. We also introduce parameters $\lambda=\frac{\Delta H}{H_{0}}=\frac{R o}{B u}$, and $d=\frac{H_{10}}{H_{20}}$. The non-dimensional background thicknesses then are $H_{1}=d_{1}$ and $H_{2}=d_{2}-\lambda e^{-y^{2}}$ (necessarily, $d_{2} \geq \lambda$ ).

We take typical values of parameters $d=1 / 4$ and $\rho=0.5$, while varying the values of $R o$ and $B u$ in what follows. As in the one-layer version of the model, all calculations below are made with non-dimensional equations.

The jet configurations in the geostrophic $(R o=0.05)$ and ageostrophic $(R o=1.5)$ cases are presented in Fig. 2. As in the one-layer case, normalized absolute vorticity is not sign-definite in the ageostrophic case, and we expect the appearance of inertial instability at $R o \geq 1 / 4$. Again, it is easy to check that the Ripa's general stability conditions for multi-layer flows ${ }^{24}$ are satisfied for $R o$ $\leq 1 / 4$, but not for $R o>1 / 4$. 

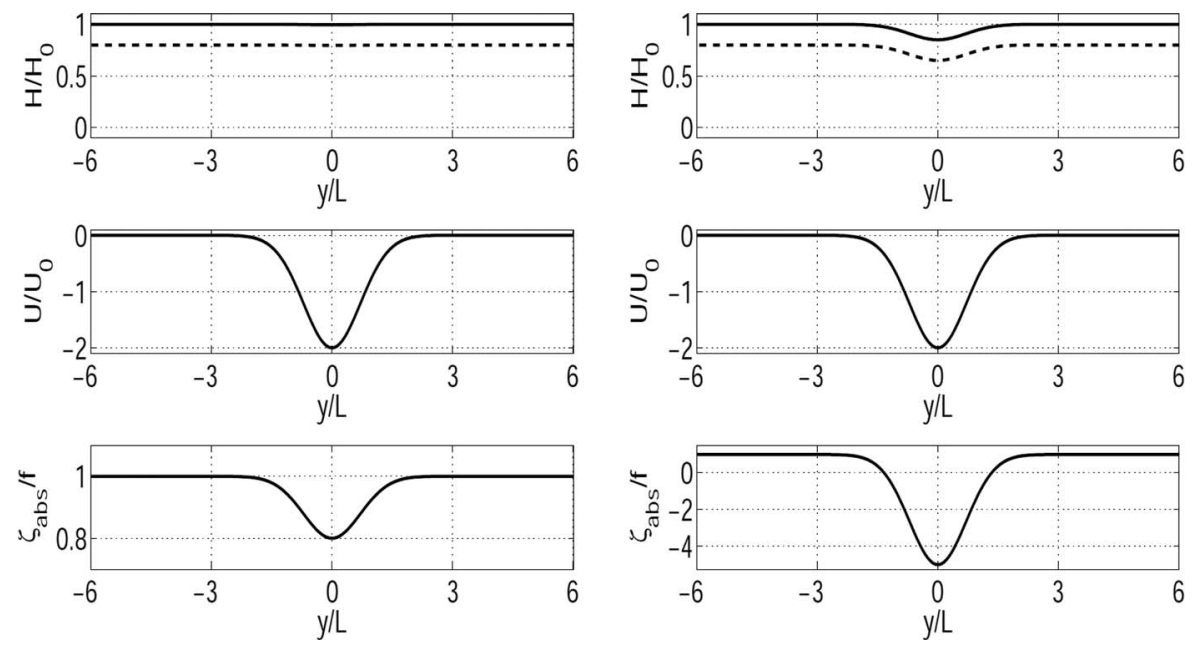

FIG. 2. Background flow with $B u=10, \rho=0.5$, and $d=0.25$ at $R o=0.05$ (left column) and $R o=1.5$ (right column). (Upper panel) thicknesses of the layers; left(right) column: $\lambda=\frac{\Delta H}{H}=\frac{R o}{B u}=5 \times 10^{-3}(0.15)$; (Middle panel) zonal velocity profile (same in both layers); (Lower panel) profile of the absolute vorticity $\beta y+\zeta$ normalized by the planetary vorticity $\beta y$ (same in both layers).

\section{RESULTS OF THE LINEAR STABILITY ANALYSIS IN THE ONE-LAYER MODEL}

\section{A. General setting: Expectations and open questions}

We consider perturbations of the background flow (2.4) in the form $\left(u^{\prime}, v^{\prime}, h^{\prime}\right)=$ $(u(y), v(y), h(y)) e^{i(k x-\omega t)}$. The linearized discretized equations are given in (3.1), where $\mathcal{D}$ denotes the Chebyshev differentiation matrix. The matrix in the r.h.s. of (3.1) is real. Therefore, if $(u(y), i v(y), h(y)) e^{i(k x-\omega t)}$ is an eigenvector associated with $(k, \omega)$, then $\left(u^{*}(y),-i v^{*}(y), h^{*}(y)\right)$ is an eigenvector associated with $(-k,-\omega)$. In what follows we will consider only the perturbations with $k \geq 0$. The results for $k \leq 0$ can be obtained by symmetry considerations. The eigenproblem (3.1) for the complex eigenvalues $\omega$ is solved at each $k$ by the pseudo-spectral collocation method ${ }^{25}$ with a typical number of the collocation points $N=250$ on the interval $(-10,10)$, in non-dimensional terms.

$$
\omega\left(\begin{array}{c}
\text { Ro } u \\
\operatorname{Ro~iv} \\
h
\end{array}\right)=\left[\begin{array}{ccc}
\operatorname{Rok} U & \left(y-\operatorname{Ro} \partial_{y} U\right) & k / \lambda \\
y & \operatorname{Rok} U & \mathcal{D} / \lambda \\
k H & -\left(\partial_{y} H+H \mathcal{D}\right) & k U
\end{array}\right]\left(\begin{array}{c}
u \\
i v \\
h
\end{array}\right) .
$$

We are looking for trapped modes and impose zero boundary conditions. Numerical convergence was systematically checked by varying $N$, and was typically achieved for $N \leq 250$. The results of this analysis are presented below in the form of (1) dispersion/stability diagrams representing the non-dimensional real part of the phase velocity $c=\omega / k$ and the non-dimensional growth rate $\operatorname{Im}(\omega)$ as functions of $k$, (2) phase-portraits of the most unstable modes, and (3) meridional crosssection of $v(y)$. The output of pseudo-spectral collocation calculations can contain non-differentiable pseudo-modes. They can be filtered out, as in the previous work, e.g., Ref. 6.

As explained in the Introduction, we are interested in the long-wave instabilities. For a zonal jet on the equatorial $\beta$-plane in the one-layer model, one expects to find the classical barotropic instability. On the mid-latitude tangent plane that is, basically, all, as it may be shown by an integral estimate that all waves trapped inside a jet are suprainertial (cf. Appendix and Ref. 26), while inertial instability is due to unstable trapped waves. ${ }^{27,6}$ As shown in Appendix A, a corresponding estimate on the equatorial $\beta$-plane does not bound the eigenfrequency squared from below and, in principle, inertial instability of the jet is not excluded already in the one-layer model. In fact, it was reported for 

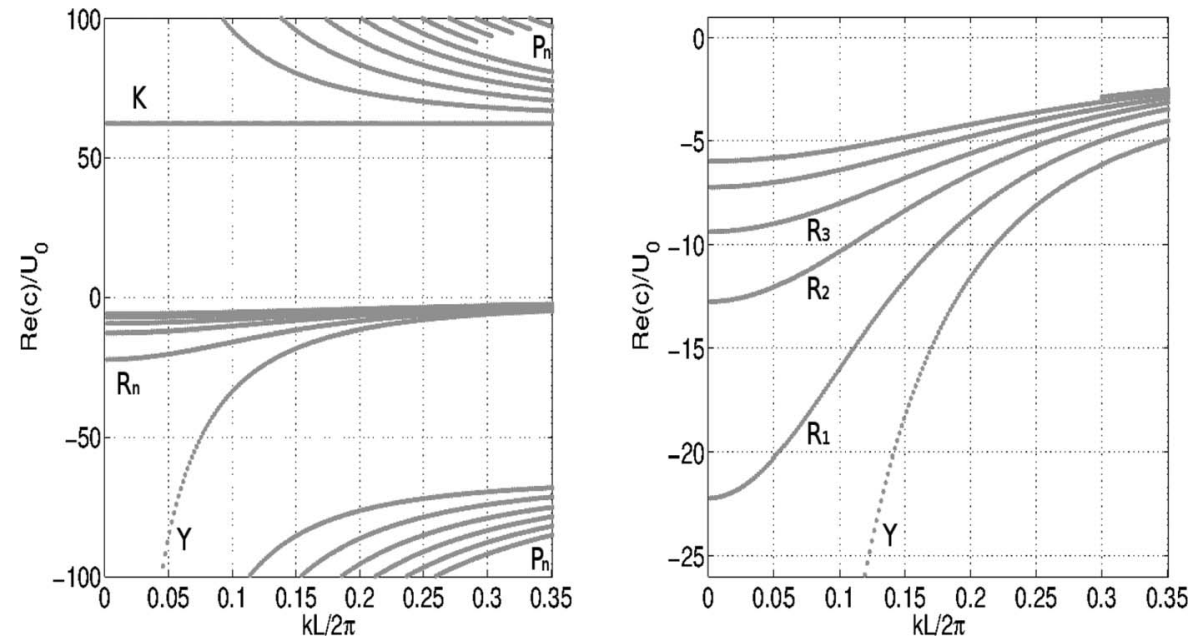

FIG. 3. Stability diagram for a background flow with $R o=0.05$ displayed in Fig. 1, left panel. (Left) stable modes. (Right) Enlarged view of the stability diagram for small $c . P_{n}$ : Poincaré (inertia-gravity) waves with meridional wavenumber $n$; K: Kelvin wave; Y: Yanai wave; $R_{n}$ : Rossby waves with meridional wavenumber $n$.

the linear shear flow in Ref. 16. So, the main question is under what conditions inertial instability is realizable in the model, and how it interplays with the barotropic instability in ageostrophic regimes.

\section{B. Increasing $R o$ at fixed $B u$}

In this subsection, we display the results for a fixed value of the Burger number $B u=10$ and the Rossby number increasing from essentially geostrophic to essentially ageostrophic values.

\section{Stable configuration: $R \mathbf{R o}=0.05$}

In this case of a small Rossby number, the jet is weak and stable. The eigenmodes are equatorial shallow-water waves with standard structure. ${ }^{12}$ Their detailed phase-portraits may be found, e.g., in Ref. 28. The dispersion diagram presented in Fig. 3 displays high-speed westward- (left-) and eastward- (right-) propagating inertia-gravity, or Poincaré waves; eastward-propagating nondispersive Kelvin wave; westward propagating low-speed dispersive Rossby waves, and dispersive westward propagating wave intermediate between Rossby and inertia-gravity ones, the Yanai wave. We should recall ${ }^{12}$ that the standard way of deducing the spectrum of equatorial waves over the state of rest is to reduce the linearized RSW system, after Fourier-transform in $x$ and $t$, to a single equation for $v(y)$ and to solve this latter with the help of decomposition in parabolic cylinder functions $D_{n}(y)$, where $n=0,1,2, \ldots$ gives the number of nodes of the respective eigenfunction. The resulting cubic dispersion relation gives two singular solutions corresponding to the Kelvin wave $(\mathrm{K})$, which can be formally associated with $n=-1$ and has no meridional velocity at all, the Yanai wave (Y) corresponding to $n=0$, and a triple solution for each $n$ starting from 1 , consisting of pair of higher frequency Poincaré waves $(\mathrm{P})$ and a lower-frequency Rossby wave $(\mathrm{R})$. In the presence of (non-uniform) mean flow this analysis does not hold, yet the number of nodes of the meridional velocity field in the meridional direction can be used to identify the waves in a robust topological way. We will use this diagnostics below. In the present case of weak mean current, the eigenmodes are close to the classical equatorial waves, which is confirmed by the structure of the velocity field and by the eigenvalues of the corresponding wave-speeds (not presented).

\section{Intermediate Rossby number configuration: $\mathbf{R o}=\mathbf{0 . 3}$}

When Rossby number of the jet increases, the instability appears beyond $R o \simeq 0.25$, consistently with Ripa's criterion. The stability diagram of Fig. 4 displays several different types of instabilities 

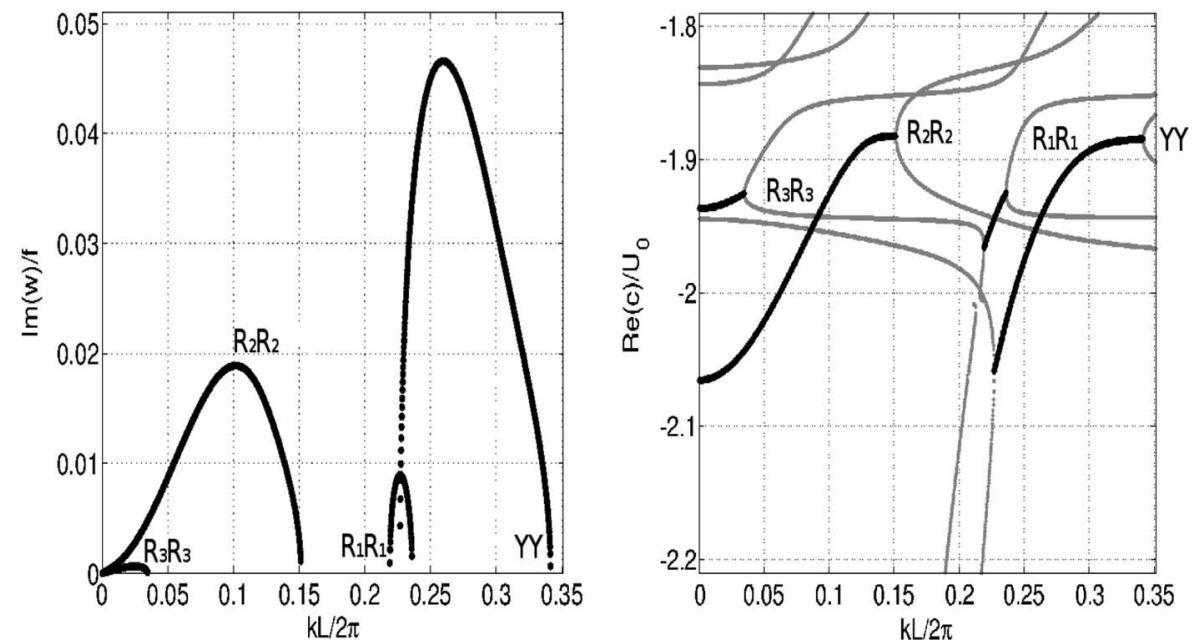

FIG. 4. Stability diagram for the background flow with $B u=10$ and $R o=0.3$. (Black) unstable modes. (Gray) stable modes. $R_{n}$ : Rossby waves; Y: Yanai wave. Here and below the growth rate is normalized by $f=\beta L$.

arising at $R o=0.3$, which may be associated with phase-locking and resonances between various types of propagating waves. Yet, with respect to the similar stability problem on the $f$-plane ${ }^{6}$ the situation is more complicated here due to the presence of multiple species of waves. The resonating wave species are identified by the number of nodes in the meridional velocity and by the phaseportraits extracted from two branches of the dispersion diagram near the intersection point. This identification, when it is unambiguous, is marked in the figure. Simultaneous presence of multiple waves species, and corresponding large number of close dispersion curves, as well as hybridization, which modifies the standard phase-portraits, complicate the identification in some cases. However, we should stress that, here and below, the number of nodes of the unstable mode never changes along the instability curve.

We should recall (e.g., Refs. 29 and 30 in the one-layer rotating shallow water model on the $f$-plane) that the resonance between two Rossby waves is associated with the standard barotropic instability. It does manifest itself in the long-wave sector in the present case of equatorial jet, although the strongest instability is due to the resonance of Yanai waves. This dominant instability shuts down at small but finite wavenumbers, unlike the classical barotropic one, cf. Fig. 4. While the unstable modes of the standard barotropic instability branch of figure are balanced, in the sense that velocity vectors follow the isobars, the unstable modes of the new instability are essentially unbalanced (not shown).

\section{High Rossby number configuration: $R=1.2$}

With increasing Rossby number the stability diagram evolves and reveals an increasing number of resonances (intersections of the dispersion curves) and related instabilities. Still the dominant ageostrophic instability due to the resonance of Yanai waves persists, with substantially increasing growth rates. We show in Fig. 5 the stability diagram at $R o=1.2$. The small-amplitude growth rates close to the $k$-axis are removed, as well as non-resonating stable modes (one can get an idea of both from the figures in Sec. III C). As before, we identify the resonating modes by considering the structure of the meridional velocity and the phase-portraits close to the intersections of the dispersion curves. The most unstable (having the maximal growth rate) mode is presented in Fig. 6. As seen from the figure, it has zero-node Yanai-wave structure of the meridional velocity and is dipolar, in what concerns the pressure perturbation, in the meridional direction. On the contrary the most unstable mode of the second instability branch in Fig. 5 is tripolar (not shown). Both structures are consistent with the nature of the corresponding resonating waves indicated in the figure. It should be noted that $R_{2} R_{2}$-instability, next-to-dominant at lower Rossby numbers, cf. Fig. 4 , gives way to the $R_{1} R_{1}$ one. 

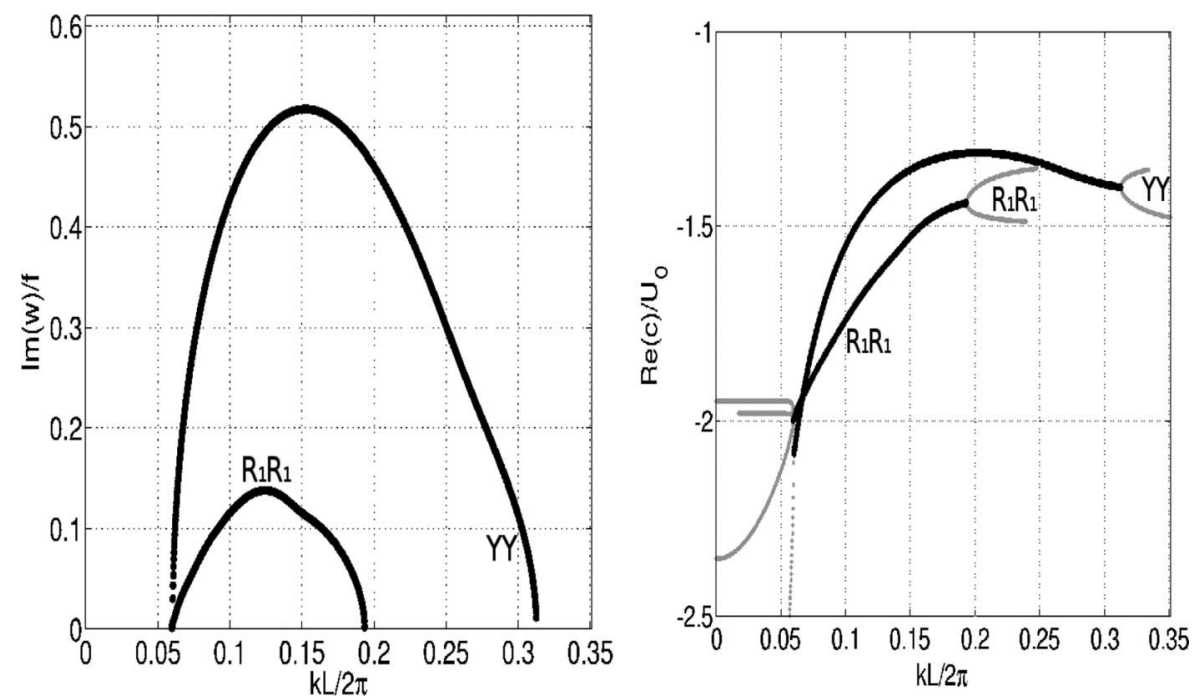

FIG. 5. Stability diagram for a background flow associated with $R o=1.2$ displayed in Fig. 1, right panel. Same conventions as in Fig. 4.

To summarize, at fixed large Burger number increasing the Rossby number destabilizes the flow, according to Ripa's criterion, with two main types of long-wave instabilities corresponding to the resonances of Rossby and Yanai waves, the second one being dominant and acting in a range of wavenumbers bounded from below.

\section{Diminishing Bu at fixed (ageostrophic) Ro}

We now choose a typically ageostrophic value of the Rossby number $R o=1$ and vary the Burger number. As may be inferred from the estimate (A3) in Appendix A, inertial instability is favored by low values $B u$, so we will diminish the Burger number with respect to Subsection III B.

In Fig. 7, we present the evolution of the stability diagram with Burger number diminishing from $B u=10$, as in Subsection III B, to $B u=2$. As is clear from the figure, the lower boundary of the instability in the wavenumber space is shifting downwards, while the maximum growth rate remains basically the same. One can thus infer that the strength of the instability is controlled by the Rossby number, while Burger number controls its range in the wavenumber space. This
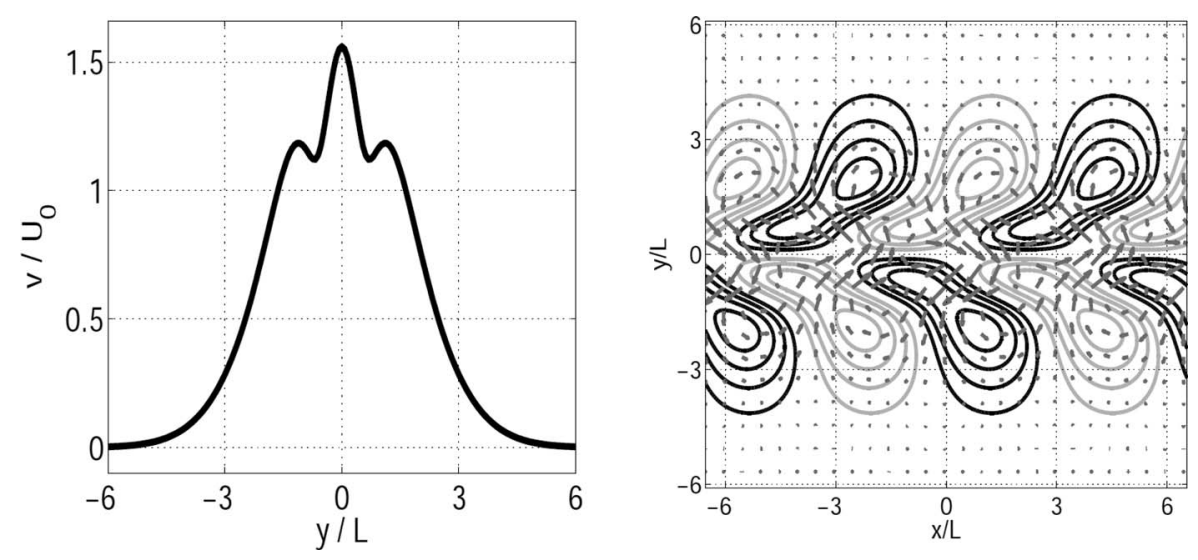

FIG. 6. (Left) The profile of $v(y)$ and (Right) two-dimensional structure of the most unstable mode with $k=0.152\left(\frac{2 \pi}{L}\right)$, $\operatorname{Re}\left(c / U_{0}\right)=-1.35$, and $\operatorname{Im}(\omega / \beta L)=0.52$ of Fig. 5. (Arrows) velocity field, contours: thickness anomaly. (Black) positive, (Gray) negative. 

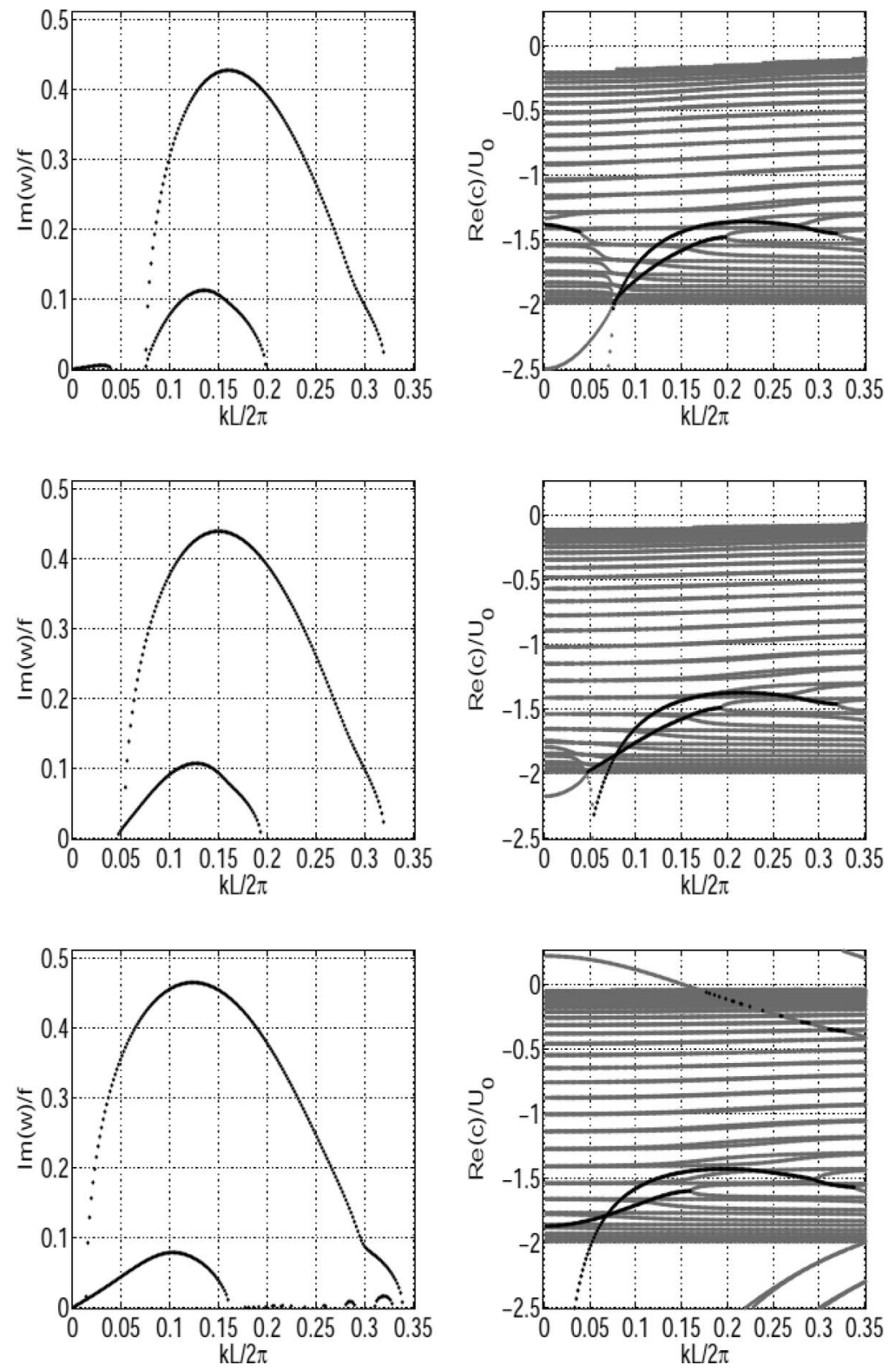

FIG. 7. Growth rates and phase speeds of the unstable modes as functions of $k$ for a background flow with $R o=1$, and $B u$ $=10$ - upper row, $B u=5-2$ nd row, $B u=3-3$ rd row, and $B u=2-$ lower row. (Black) unstable modes, (Gray) stable localized solution of (3.1). Upper curve in the left column: $Y Y$-instability.

tendency is confirmed by further diminishing $B u$, as shown in Figure 8. Moreover, at low enough $B u$ the instability curve hits zero in $k$ at nonzero growth rate. This is the classical symmetric inertial instability.

Yet, as was already observed in the two-layer model on the $f$-plane,${ }^{6}$ the corresponding asymmetric instability is still stronger. The meridional structure of the most unstable mode is presented in Fig. 9, right panel, together with the most unstable modes for higher values of $B u$. The meridional structure of the symmetric unstable mode $(k=0)$ is very similar (not shown). This structure (no nodes) is consistent with the interpretation of symmetric instability mode as a trapped eigenmode with lowest eigenvalue. The no-node structure explains a posteriori the relation of this instability to $Y Y$-resonances, as Yanai waves have, precisely, the required meridional structure. Although the 

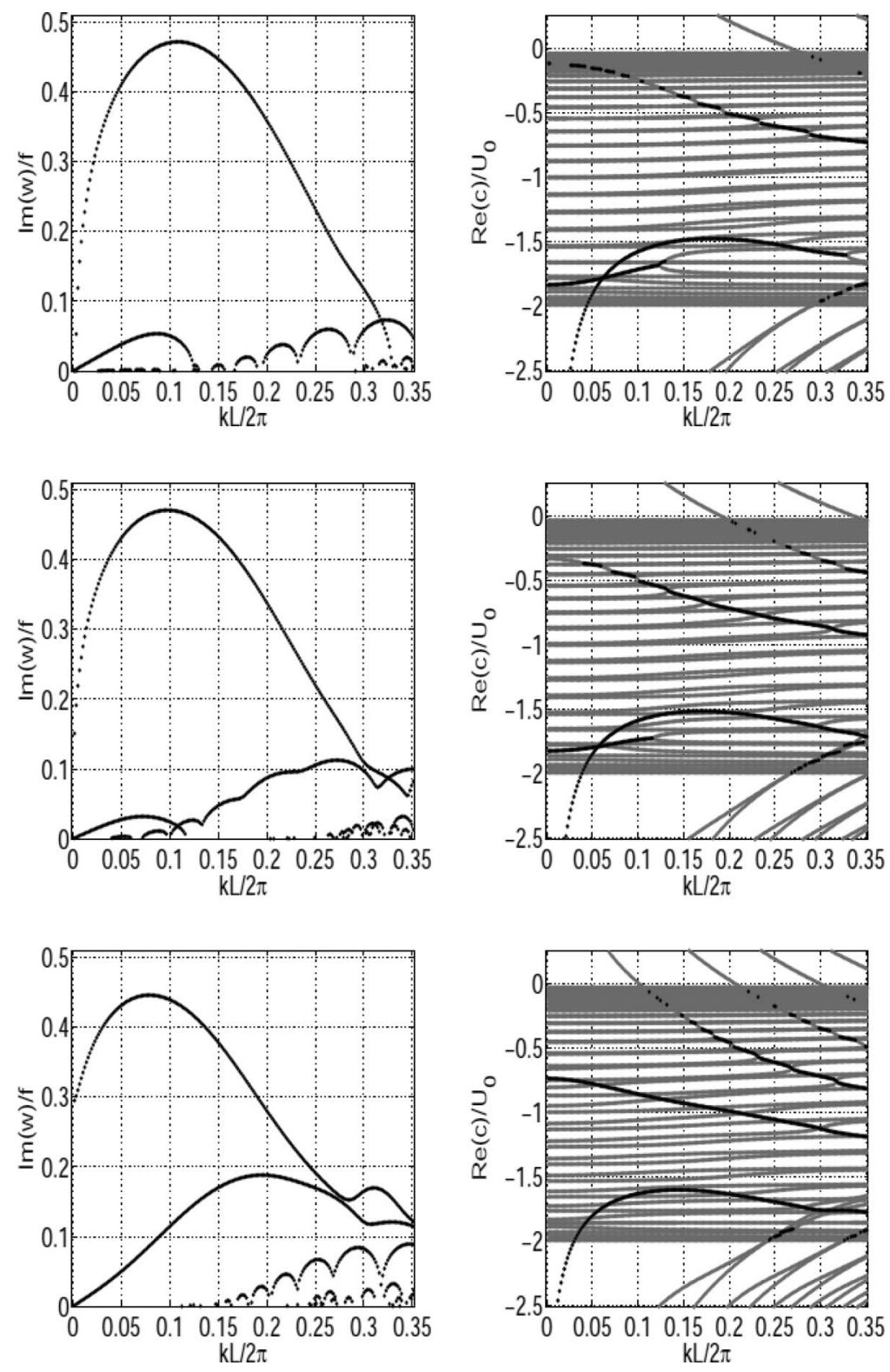

FIG. 8. Growth rates and phase speeds of the unstable modes as functions of $k$ for a background flow with $R o=1$, and $B u=$ 1.5 - upper row, $B u=1.3$ - 2nd row, $B u=1.1$ - 3rd row. (Black) unstable modes, (Gray) stable localized solution of (3.1). Upper curve in the left column: $Y Y$-instability.

meridional velocity is strongly localized, cf. Fig. 9, its profile is non-singular at $y=0$, as follows from the zoom of the figure (not shown).

One cannot significantly diminish further the value of the Burger number due to incropping of the free surface at the center of the jet. We observe a tendency to flattening of the growth-rate curve in the vicinity of $k=0$ for the values of $B u$ close to the incropping threshold (not shown), although the asymmetric instability is always the strongest.

To summarize, at fixed ageostrophic value of the Rossby number and diminishing Burger number, the leading $Y Y$-instability develops a non-zero growth-rate limit at $k=0$ giving the standard symmetric inertial instability, while keeping the maximal growth rate at small but non-zero $k$. 

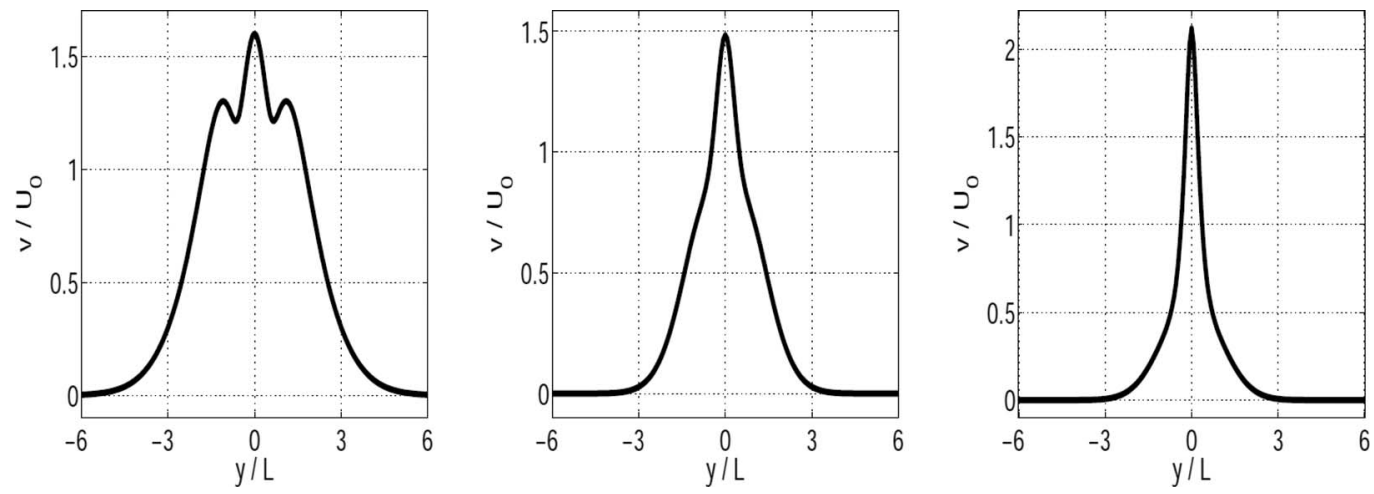

FIG. 9. The profile of $v(y)$ for the most unstable mode of the background flow with $R o=1$, and $B u=10-$ (left), $B u=1.5$ - (middle), $B u=1.1-$ (right).

\section{A résumé of the linear stability analysis in the one-layer model}

Thus, our analysis reveals that, in the framework of the one-layer shallow water model on the equatorial beta-plane, Gaussian easterly jets centered at the equator are stable at small Rossby numbers and become unstable as Rossby number exceeds $1 / 4$. The standard barotropic instability of the jet is present, but is dominated by an ageostrophic instability arising from phase-locking and resonance between Yanai waves propagating on the background of the jet. At small enough Burger numbers this leading instability has a non-zero growth rate at $k=0$, thus giving the classical symmetric inertial instability. Yet the asymmetric inertial instability is dominant through the whole range of parameters we investigated.

\section{RESULTS OF THE LINEAR STABILITY ANALYSIS IN THE TWO-LAYER MODEL}

\section{A. General setting: Expectations and open questions}

As in Sec. III, we consider perturbations of the background flow (2.9) in the form $\left(u_{i}^{\prime}, v_{i}^{\prime}, h_{i}^{\prime}\right)=\left(u_{i}(y), v_{i}(y), h_{i}(y)\right) e^{i(k x-\omega t)}$, and write down the linearized equations (4.1), which are non-dimensionalized, discretized and where $\mathcal{D}$ denotes the Chebyshev differentiation matrix. The matrix in the r.h.s. of (4.1) is real. Again, by symmetry considerations, we limit ourselves by $k$ $\geq 0$, and seek the eigenvectors of (4.1) with complex eigenvalues $\omega$ by the collocation method.

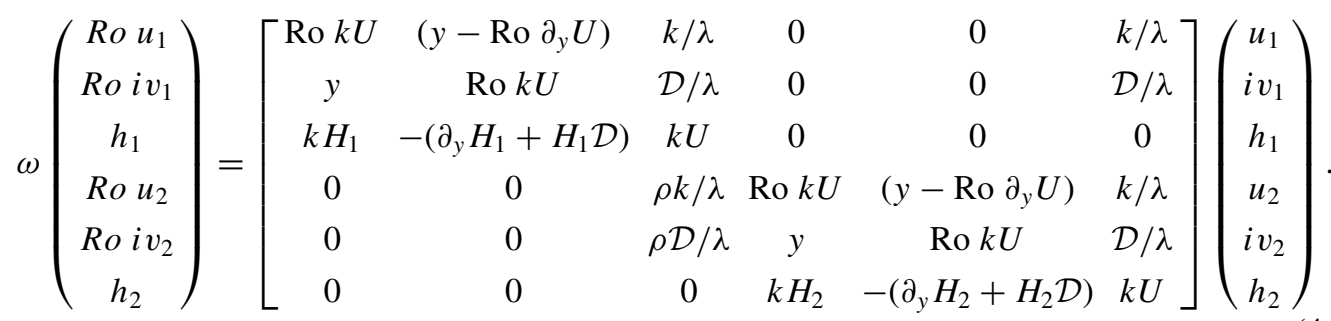

In this simplest possible baroclinic model, we expect the appearance of both baroclinic and barotropic instabilities related to resonances between barotropic and baroclinic waves, respectively. We can also expect the appearance of the baroclinic inertial instability, as it manifests itself already on the $f$-plane as the long-wave limit of ageostrophic baroclinic instability. ${ }^{6}$ The experience of Sec. III teaches us that barotropic symmetric inertial instability may be also expected. Questions then arise about relative strength of these instabilities, their relation to the asymmetric counterparts, and their zone of residence in the parameter space. 

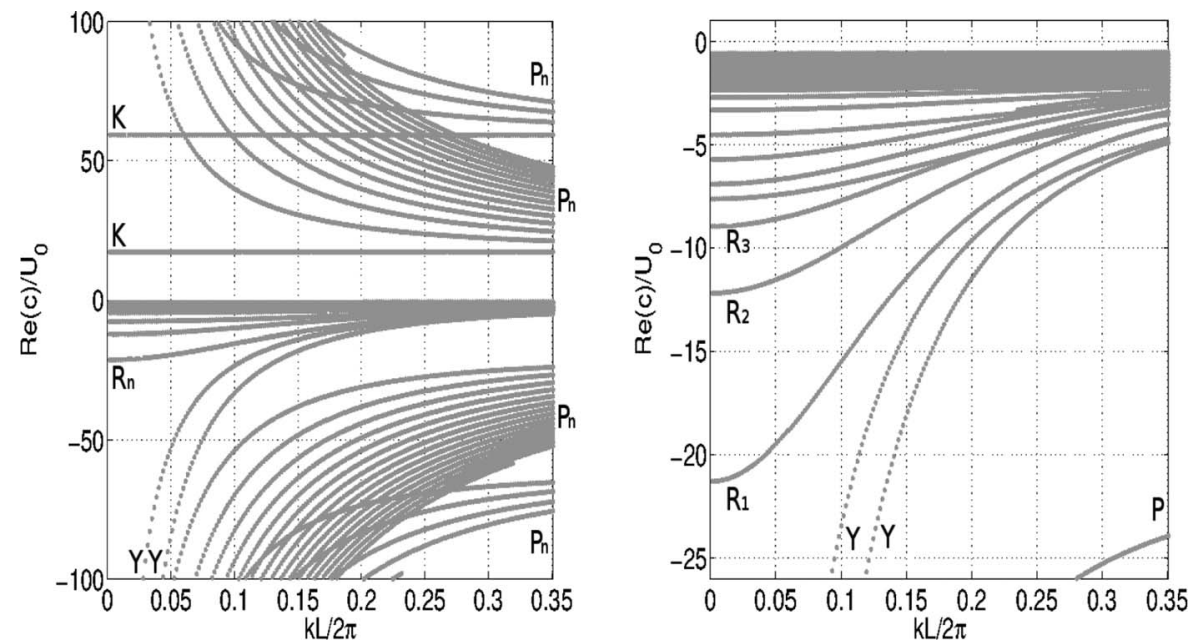

FIG. 10. Stability diagram for the background flow with $R o=0.05$ displayed in Fig. 2, left panel. (Left) barotropic and baroclinic stable modes; (Right) Enlarged view of the stability diagram at small $c . P_{n}$ : Poincaré (inertia-gravity) waves; $K$ : Kelvin wave; $R_{n}$ : Rossby waves; $Y$ : Yanai waves.

\section{B. Increasing $R o$ at fixed $B u$}

As in the one-layer case, we start wit fixing the Burger number at 10 and increasing the Rossby number.

\section{Stable configuration: $\mathbf{R o}=0.05$}

We begin with the stable configuration with small Rossby number $R o=0.05$. The corresponding dispersion diagram of Fig. 10 clearly displays (less obviously for the Rossby waves accumulating near the $k$-axis) the doubling of the wave-species observed in the one-layer case, cf. Fig. 3: one slower and one faster wave of each kind. They, naturally, correspond to the baroclinic and the barotropic waves of each kind. Analysis of the meridional structure of velocity, and of the phase-portraits of different wave-species corresponding to various branches of the dispersion curves of Fig. 10 confirms this diagnostics (not presented).

We should emphasize that an intersection of dispersion curves is a necessary, but not a sufficient condition for phase-locking and resonance leading to instability. The waves with close frequencies should be having opposite intrinsic frequencies (counterpropagating with respect to the flow) and be coupled through pressure perturbations, cf. Ref. 31, in order to produce an instability. The intersections of Fig. 10 are "harmless" in this sense.

\section{Configuration close to the instability threshold: $R o=0.27$}

As in the barotropic one-layer case, the instability appears beyond $R o \simeq 0.25$. We show in Fig. 11 the stability diagram for $R o=0.27$. As seen from the right panel of Fig. 11, the dispersion curves and the instability zones are rather entangled. Yet, by analyzing the meridional structure of velocity and phase-portraits of the resonating modes we manage to identify the origin of the instabilities. Thus, two dominant instabilities at $k \approx 0.27\left(\frac{2 \pi}{L}\right)$ arise from the resonance between the pairs of the barotropic (the rightmost curve in the left panel of Fig. 11) and of the baroclinic (the next one) Yanai waves (YY-instability).

If one moves from higher towards lesser $k$ on the stability diagram on the left panel of Fig. 11, the weaker instabilities are produced by the resonances of the following waves (in order): barotropic $R_{1} R_{1}$; baroclinic $R_{1} R_{1}$, barotropic $R_{2} R_{2}$, baroclinic $R_{2} R_{2}$, and finally barotropic $R_{3} R_{3}$. The situation is, thus close in what was observed in Sec. III B 2 for the one-layer model with doubling of the instabilities of each kind, which may be barotropic or baroclinic. It is worth noting that the baroclinic YY-instability is slightly stronger than its barotropic counterpart for the given values of parameters. 

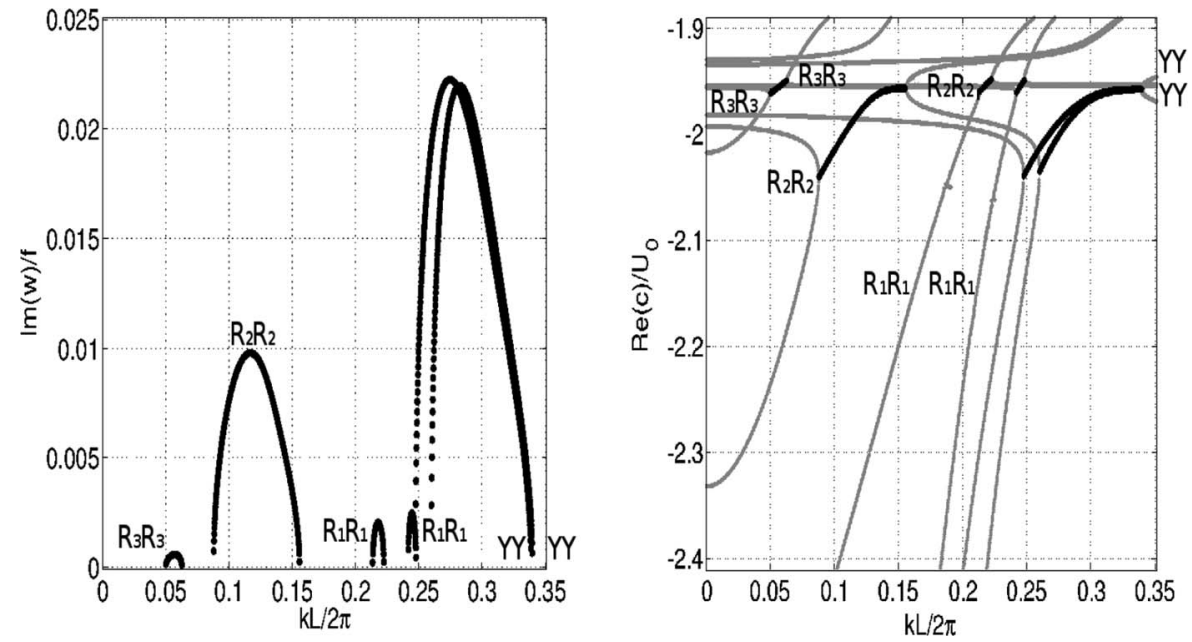

FIG. 11. Stability diagram for a background flow with $B u=10, \rho=0.5, d=0.25$, and $R o=0.27$. (Black) unstable modes. (Gray) stable modes. $R_{n}$ : Rossby waves; Y: Yanai waves.

We will see below that this feature will be more and more pronounced with increasing Rossby number. We should also stress that although the Rossby number is rather small, the domain of the leading instabilities is separated from zero in the wavenumber space, unlike the classical baroclinic and barotropic instabilities on the $f$-plane.

In Figs. 12 and 13, we give an example of the unstable modes corresponding to the two dominant instabilities at the higher- $k$ end of the left panel of Fig. 11, not far from the intersection of the dispersion curves on the right panel. The characteristic structure of the, respectively, baroclinic and barotropic Yanai waves is clearly recognizable in the figures.

\section{Higher Rossby numbers and appearance of the symmetric inertial instability}

With increasing Rossby numbers the dispersion diagrams become more and more complicated. Numerous instabilities appear due to the intersections of the entangled dispersion curves. We do not seek to resolve all the instability modes and will concentrate our attention on the dominant instabilities. In Fig. 14, we display the stability diagrams for the values $0.5,0,8,1.2$, and 1.5 of the Rossby number. Two instability modes are clearly dominant. They correspond to the barotropic
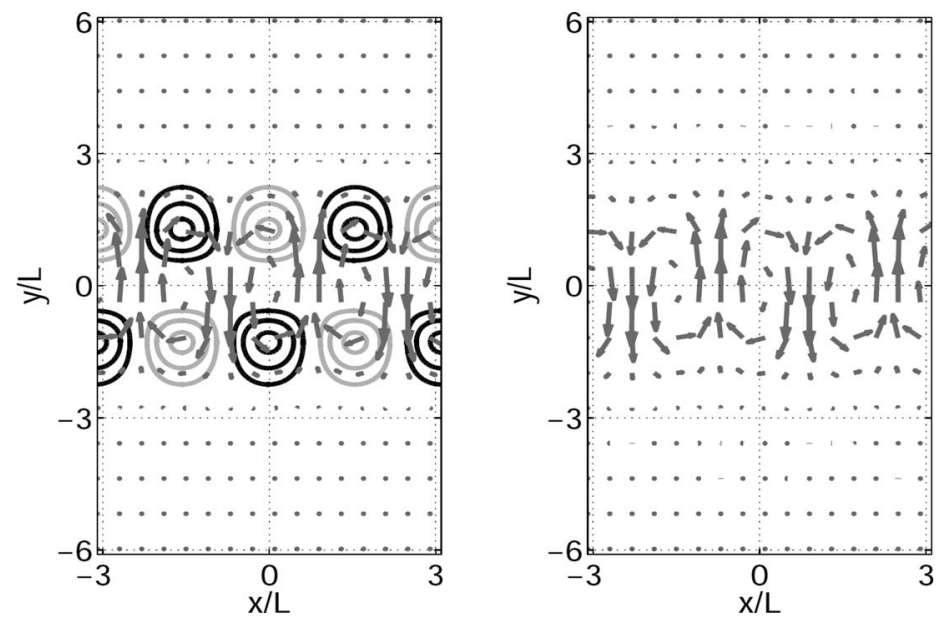

FIG. 12. Two-dimensional structure of the unstable mode with $k=0.32\left(\frac{2 \pi}{L}\right)$ and $\operatorname{Re}\left(c / U_{0}\right)=-1.943, \operatorname{Im}(\omega / \beta L)=0.0028$ of Fig. 11. Same convention as in Fig. 6. (Left) upper layer, (Right) lower layer. 

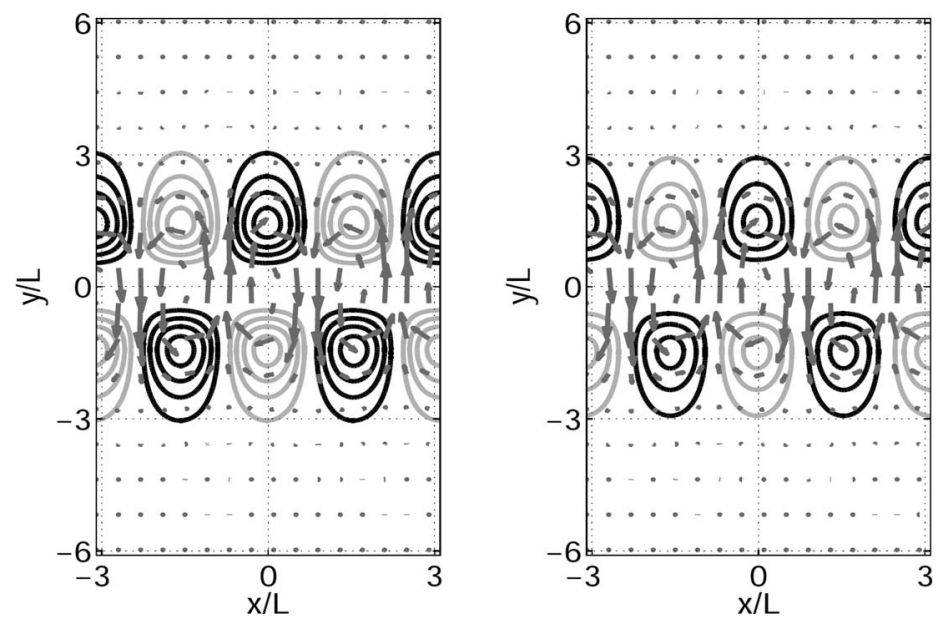

FIG. 13. Two-dimensional structure of the unstable mode with $k=0.32\left(\frac{2 \pi}{L}\right)$ and $\operatorname{Re}\left(c / U_{0}\right)=-1.945, \operatorname{Im}(\omega / \beta L)=0.0049$ of Fig. 11. Same convention as in Fig. 12.

(rightmost upper curve) and baroclinic (leftmost upper curve) YY-instabilities identified at $R o=0.27$ above. With increasing $R o$ both curves shift towards smaller $k$. At high enough Ro, i.e., for essentially ageostrophic configurations, the curve of the baroclinic $Y Y$-instability crosses the ordinate axis, thus giving a symmetric instability with $k=0$. This instability is a classical equatorial inertial instability, which we checked by performing a separate stability analysis in the symmetric case $k \equiv 0$ (not
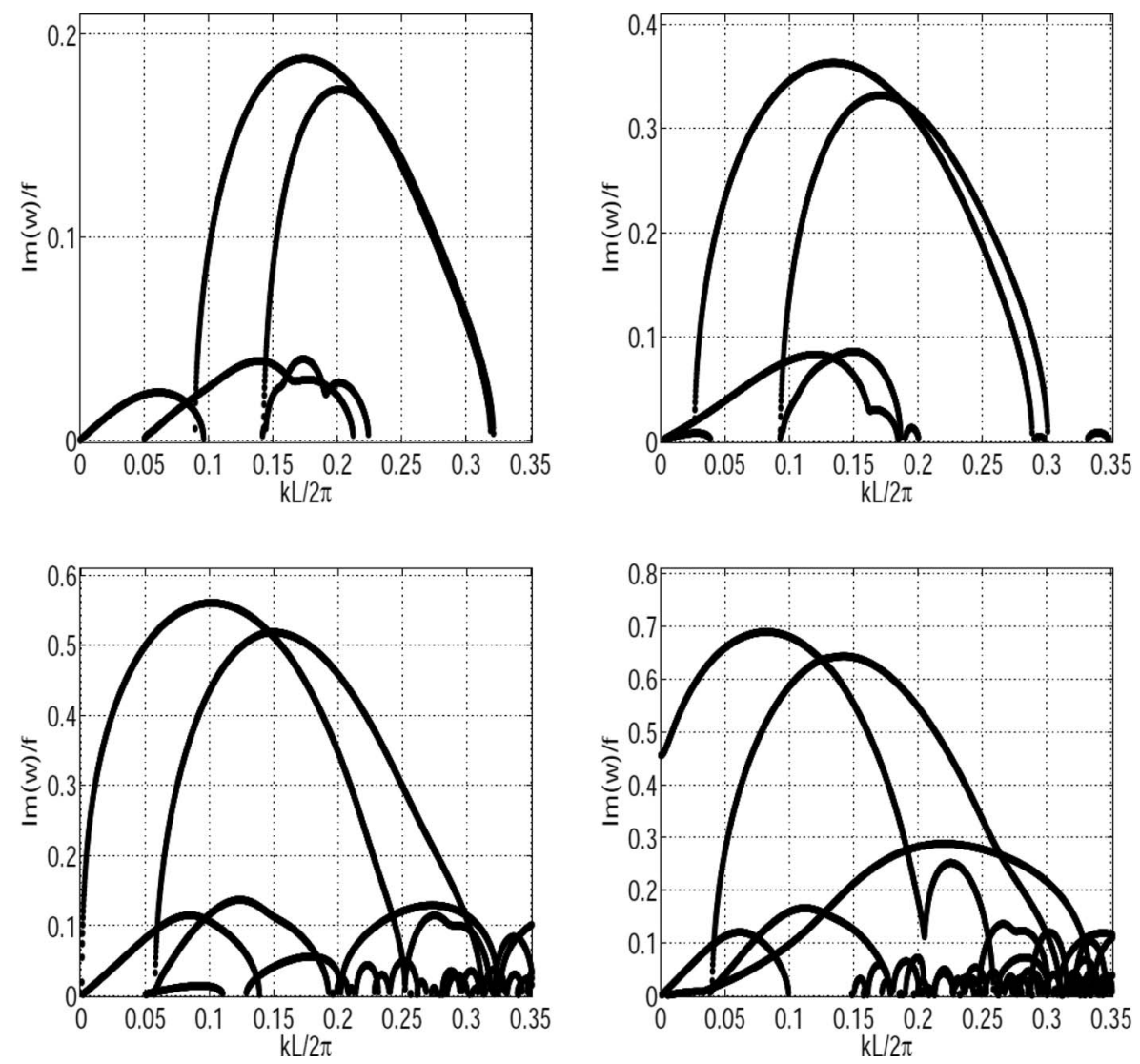

FIG. 14. Growth rates of the unstable modes as functions of $k$ for a background flow with $B u=10, \rho=0.5, d=0.25$, and $R o=0.5$ - upper left, $R o=0.8-$ upper right, $R o=1.2$ - lower left, and $R o=1.5$ - lower right. (Black) unstable modes. 

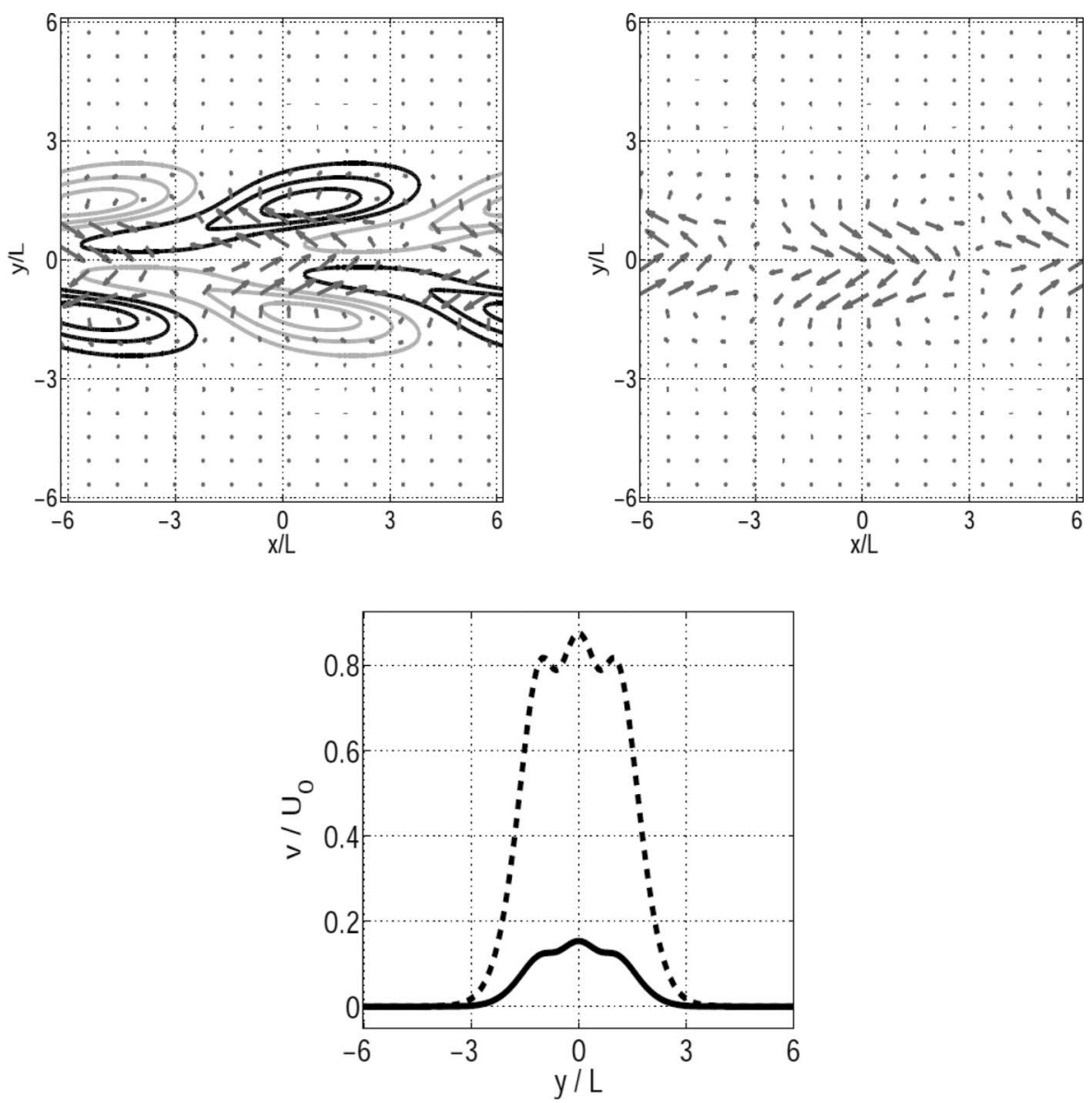

FIG. 15. (Top) Two-dimensional structure of the unstable mode with $k=0.08\left(\frac{2 \pi}{L}\right)$ and $\operatorname{Re}\left(c / U_{0}\right)=-1.37, \operatorname{Im}(\omega / \beta L)=$ 0.69 of $R o=1.5$ (Fig. 14). Same conventions as in Fig. 12. (Bottom) Profile of the meridional velocity $v(y)$. (Dashed) upper layer; (Continuous) lower layer.

presented). We, thus, observe the same phenomenon as in the $f$-plane configuration: ${ }^{6}$ the symmetric inertial instability is a $k \rightarrow 0$ limit of the ageostrophic baroclinic instability, yet the maximum growth rate is achieved for small, but non-zero $k$ (asymmetric inertial instability ${ }^{32}$ ). The structure of the corresponding most unstable mode is presented in Fig. 15 and confirms its YY-origin.

\section{Diminishing Bu at fixed (ageostrophic) Ro}

We now fix the value of $R o=1$, in order to be in the ageostrophic regime where inertial instability is operational, and diminish $\mathrm{Bu}$. Figures 16 and 17 present the corresponding evolution of the stability diagam.

As follows from the figures, the baroclinic (the stronger one in Fig. 16) YY-instability hits the ordinate axis at low enough $B u$ producing non-zero growth rate at zero $k$, and thus a symmetric instability. The barotropic YY-instability is also shifting towards lower $k$, but another instability branch corresponding to the baroclinic $R_{1} R_{1}$ resonance starts intensifying at low $k$ and hits the axis in its turn. Then, it is the barotropic YY-instability which does the same. Still, the asymmetric versions of each instability remain dominant. The meridional structure of the most unstable modes of the three leading branches at three different values of $B u$ is presented in Fig. 18. The meridional structure of their symmetric $(k=0)$ counterparts is very close, indicating that higher meridional modes of the symmetric inertial instability make their appearance at low enough $B u$. This is completely consistent with the interpretation of the symmetric instability in terms of trapped modes with negative 

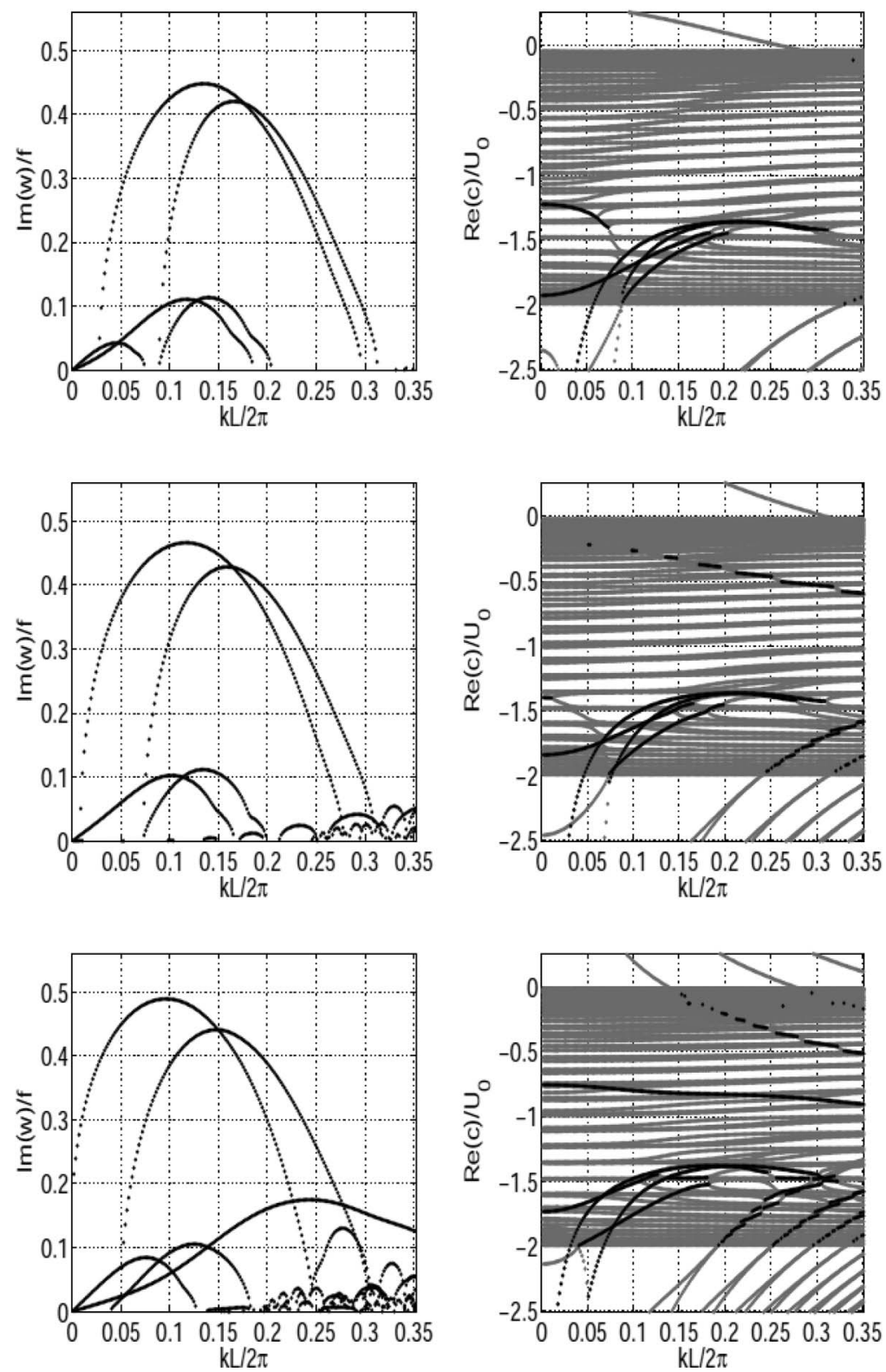

FIG. 16. Growth rates and phase speeds of the unstable modes as functions of $k$ for a background flow with $R o=1$, and $B u$ $=20-1$ st row, $B u=10-2$ nd row, and $B u=5-3$ rd row. (Black) unstable modes, (Gray) stable localized solutions of (4.1). Two upper curves in the left column: baroclinic (the higher) and the barotropic $Y Y$ - instabilities.

eigenfrequency squared: the gravest mode, according to the Sturm theorem, has no nodes, the next one has one node, etc. If the trapping potential defined by the background flow is deep enough, both the gravest and the next trapped modes may have the eigenvalues of $\omega^{2}$ so low that they become negative, which is what we observe in Fig. 18.

\section{Résumé of the linear stability analysis in the two-layer model}

Our analysis shows that in the framework of the two-layer shallow water model, the barotropic Gaussian easterly jet on the equatorial beta-plane is stable at small Rossby numbers but loses stability at $R o \simeq 0.25$. The dominant instabilities of the jet are produced by phase-locking and resonances between a pair of barotropic, or a pair of baroclinic Yanai waves propagating on the background of 

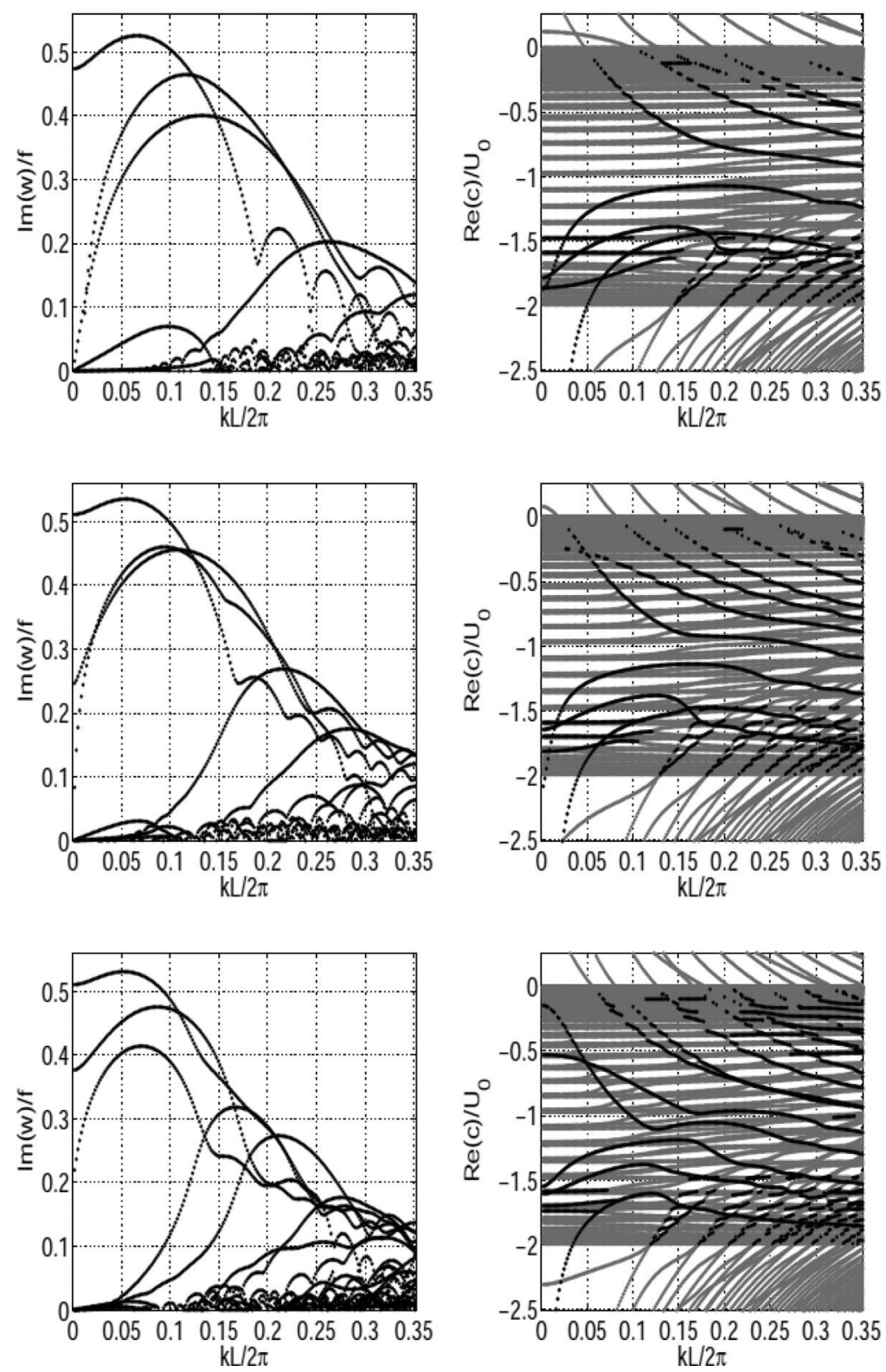

FIG. 17. Growth rates and phase speed of the unstable modes as functions of $k$ for a background flow with $R o=1$, and $B u=2-1$ st row, $B u=1.5-2$ nd row, and $B u=1.3-3$ rd row. (Black) unstable modes, (Gray) stable localized solutions of (4.1). Upper curve in the left column: baroclinic $Y Y$-instability. Second curve from the top in the left column: barotropic $Y Y$-instability (upper row), baroclinic $R_{1} R_{1}$-instability (bottom row).

the jet. The standard barotropic and baroclinic instabilities produced by the resonances of Rossby waves are also present, but are weaker. With increasing Rossby number at fixed Burger number the baroclinic YY-instability overcomes the barotropic one, and the wavenumber of the most unstable mode diminishes. At high enough $R o$ this instability has nonzero growth rate at $k=0$, thus giving a baroclinic symmetric inertial instability. Yet the maximal growth rate is achieved at small but non-zero $k$. At high enough Rossby number and diminishing Burger number the barotropic YY instability also acquires a non-zero growth rate at $k=0$ and gives rise to the barotropic symmetric inertial instability. The same phenomenon takes place with the baroclinic $R_{1} R_{1}$ instability, which thus produces the second meridional mode of symmetric instability. We should emphasize that a swap between barotropic $Y Y$ and baroclinic $R_{1} R_{1}$ instabilities takes place for low enough $B u$, the 

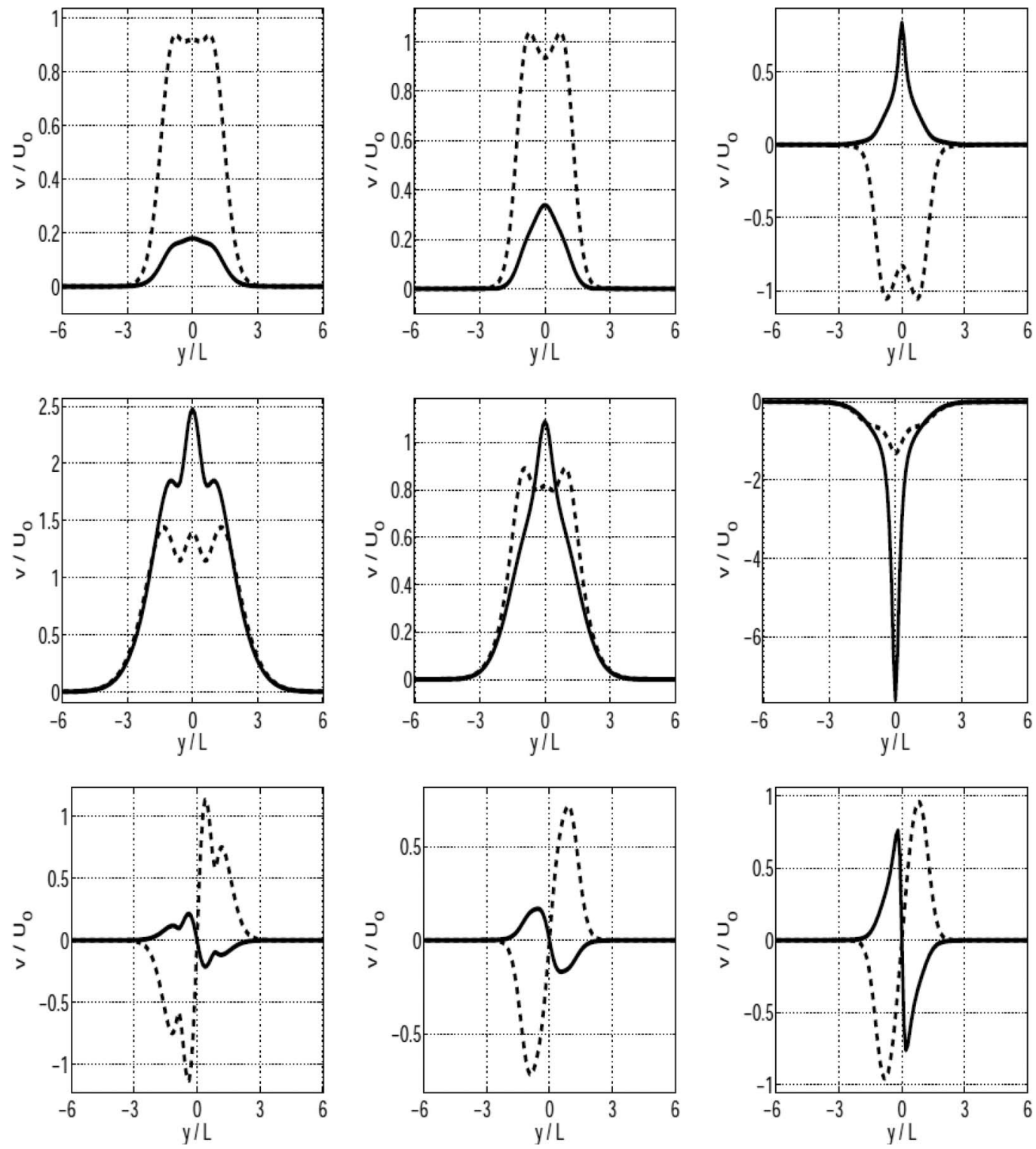

FIG. 18. Profiles of the meridional velocity of the most unstable modes of the background flow with $R o=1, \rho=0.5, d$ $=0.25$, and $B u=5-$ (left), $B u=2-$ (middle), $B u=1.3$ - (right). (Upper row) baroclinic $Y Y$-instability, (Middle row) barotropic $Y Y$-instability, (Bottom row) - baroclinic $R_{1} R_{1}$-instability.

latter instability becoming second in strength. At all values of parameters that we investigated, the asymmetric instabilities of any kind remained stronger than their symmetric fellows.

\section{NONLINEAR SATURATION OF INSTABILITIES}

\section{A. General settings}

In this section, we undertake direct numerical simulations of the nonlinear saturation of the dominant instabilities in the a geostrophic regime, both in one-layer and in two-layer configurations. We use the recent well-balanced finite-volume numerical scheme for rotating shallow water, ${ }^{33}$ and its generalization to the two-layer case. ${ }^{13}$ We initialize the simulations with the background jet with superimposed small perturbation corresponding to an unstable mode (the most unstable, or the second-unstable) identified by the linear stability analysis, of amplitude $\sim 1 \%$ of the mean flow. We 
$t=6 / \beta L$

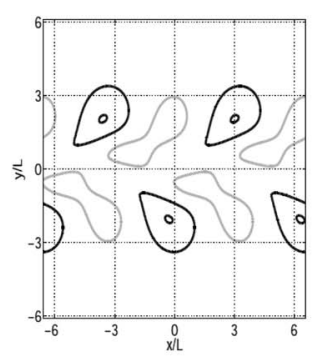

$t=20 / \beta L$

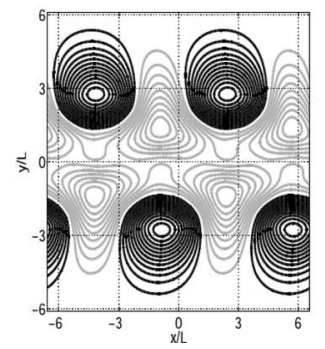

$t=8 / \beta L$

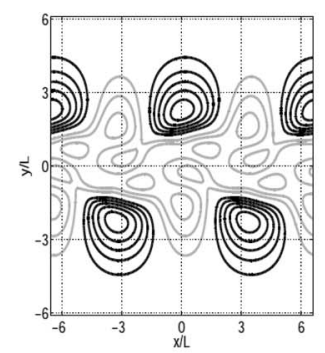

$t=24.5 / \beta L$

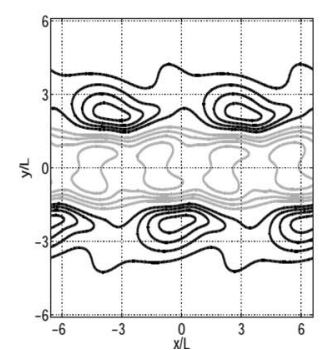

$t=11.5 / \beta L$

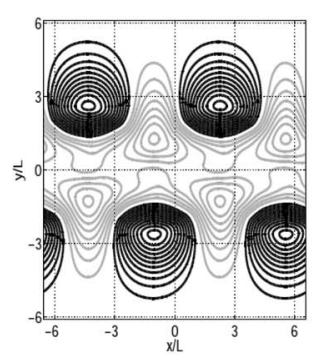

$$
t=28.5 / \beta L
$$

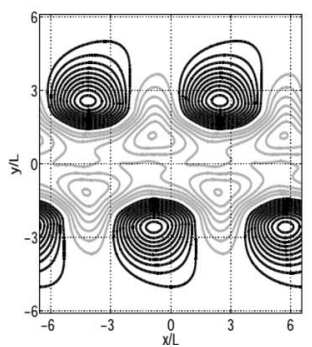

$t=16.5 / \beta L$

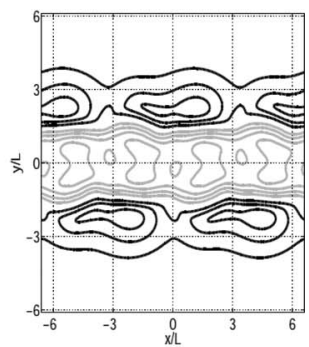

$t=34 / \beta L$

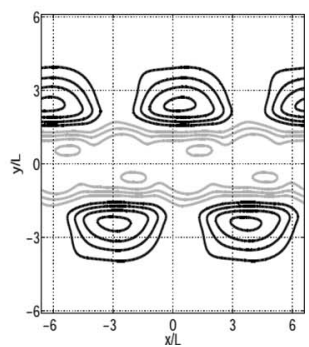

FIG. 19. Nonlinear evolution of the most unstable mode of Fig. 6 (see Fig. 5). Isolines of the normalized deviation of the thickness from its initial value. Contour interval $0.1 \Delta H$, starting at the value $\pm 0.1 \Delta H$ (positive/negative thickness anomaly: black/gray lines).

consider the configuration with $B u=10$. In the one-layer configuration we take $R o=1.2$. In the two-layer configuration we take $d=0.25, \rho=0.5$, and $R o=1.5$.

The boundary conditions are periodic (with the double of the period of the perturbation) in the along-flow direction, and sponges are used at $y= \pm 7.5$ and $y= \pm 10$, respectively, in the one- and twolayer cases, in the cross-flow direction (in non-dimensional terms). The results presented below were obtained with the (non-dimensional) spatial resolution 1/20. Control simulations with different spatial resolutions and sizes of the domain were routinely made to check numerical convergence (not shown). No explicit dissipation is introduced in the numerical scheme, numerical dissipation acting only in the zones of strong gradients (shocks) in the one-layer version, and in the zones of hyperbolicity loss in the two-layer version. The hyperbolicity loss in the two-layer model corresponds, physically, to strong shears between the layers and related Kelvin-Helmholtz type instabilities. ${ }^{34}$ Mathematically, it corresponds to the negative discriminant of the characteristic matrix of the system. The nonhyperbolic zones remain limited in space in all calculations (not shown).

\section{B. One-layer configuration with $R o=1.2$}

In Figs. 19 and 20, we present the evolution of the thickness and of the relative vorticity during the nonlinear evolution of the most unstable mode of Fig. 5. A surprising feature of Fig. 19 is a periodicity of the thickness anomaly pattern with the period $\approx 8 / \beta L$. This oscillation is also clearly seen in the evolution of the energy presented in Fig. 21. By changing the resolution and the size of the domain, we carefully checked that this oscillation is not a numerical artifact. Yet, we have no physical explanation of this manifestly nonlinear phenomenon.

The evolution of vorticity shows the transformation of the perturbation into a vortex street, which is a rather standard scenario of development of the jet instabilities. The energy evolution of Fig. 21 displays a very good overall conservation of energy, corresponding to the absence of shocks, and periodic exchanges between kinetic and potential energy corresponding to the above-discussed oscillation. Finally, we found (not shown) that non-linear saturation of the next to dominant instability is also associated with a vortex street formation and similar oscillation feature. 
$t=6 / \beta L$

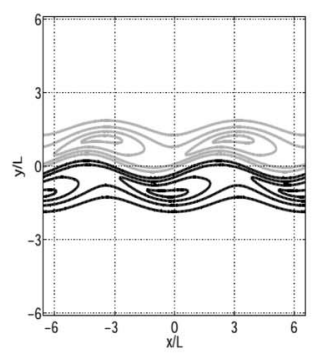

$t=20 / \beta L$

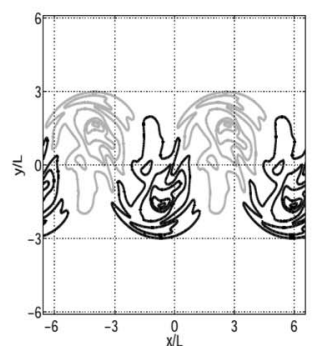

$t=8 / \beta L$

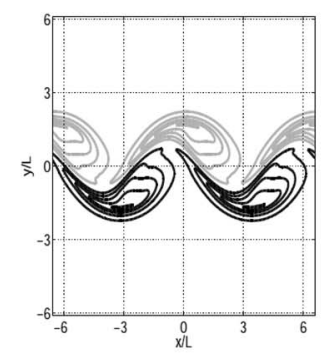

$t=24.5 / \beta L$

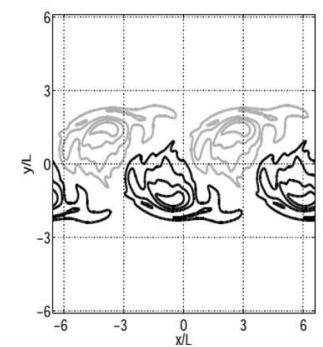

$t=11.5 / \beta L$

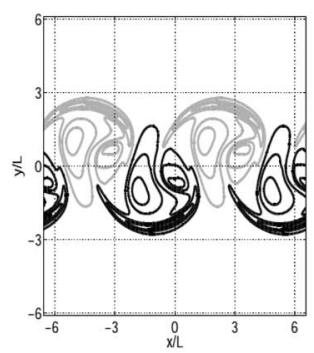

$t=28.5 / \beta L$

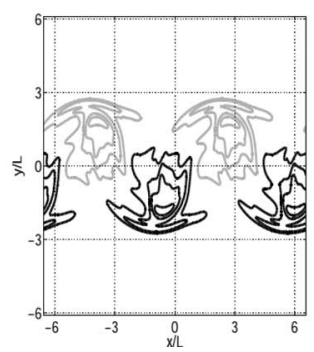

$t=16.5 / \beta L$

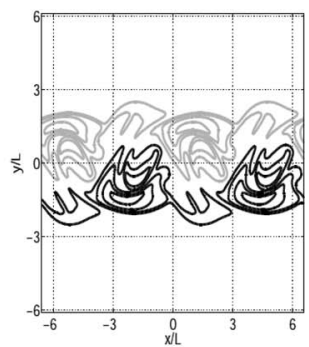

$t=34 / \beta L$

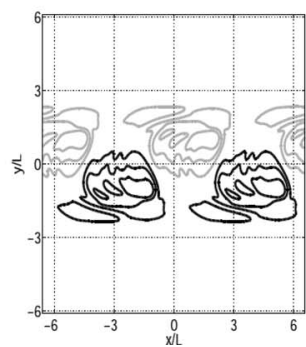

FIG. 20. Nonlinear evolution of the most unstable mode of Fig. 6 (see Fig. 5). Isolines of the relative vorticity. Contour interval $0.5 R o \beta L$, starting at the value $\pm 0.5 R o \beta L$ (positive/negative relative vorticity anomaly: black/gray lines).

\section{Two-layer configuration with $R 0=1.5$}

As was shown in Sec. IV, the dominant YY-instability duplicates in the two-layer case, as compared to the one-layer case, one instability corresponding to the resonance between barotropic, and another one - to the resonance between baroclinic waves. We made simulations of the nonlinear saturation of the most unstable modes of both instabilities. The saturation of the barotropic instability goes along the same lines as in the one-layer case of Sec. V B layerwise (not shown), with the same characteristic nonlinear oscillation. No loss of hyperbolicty was observed throughout the simulation. On the contrary, the saturation of the baroclinic instability is different. We present in Fig. 22 the corresponding evolution of the relative vorticity in the upper and lower layer.

While in the lower layer, a tendency to form a vortex street is rather clear, the evolution of the upper layer displays a lot of mixing and small-scale structures. Strong mixing and dissipation related

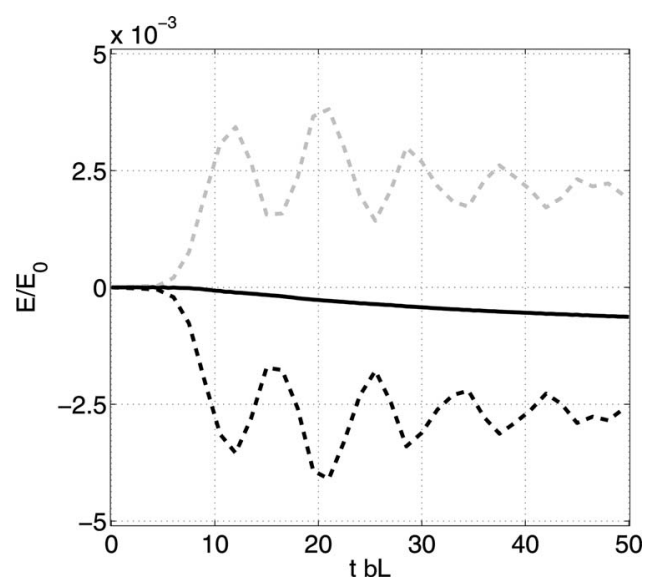

FIG. 21. Nonlinear evolution of the most unstable mode of Fig. 6 (see Fig. 5). Normalized deviation of the total (black), kinetic (black dashed), and potential (gray dashed) energy in the whole calculation domain from their initial values. 


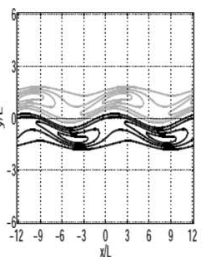

$t=5 / \beta L$

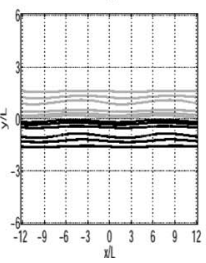

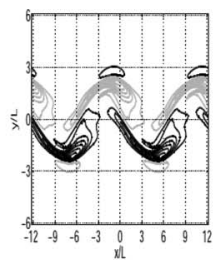

$t=7 / \beta L$

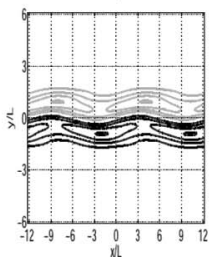

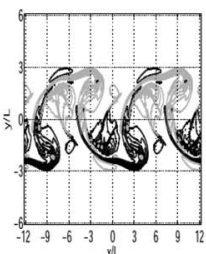

$t=10 / \beta L$

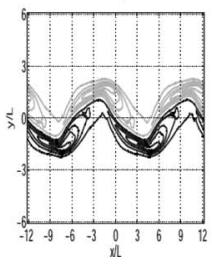

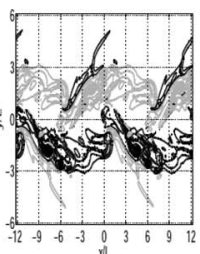
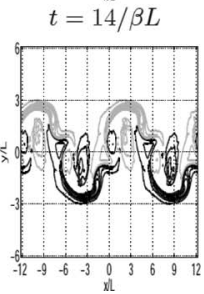

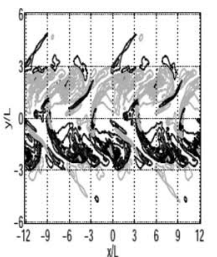

$t=19 / \beta L$

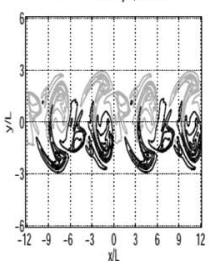

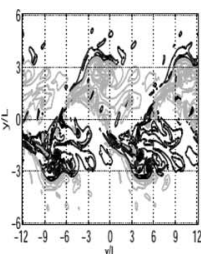

$t=28.5 / \beta L$

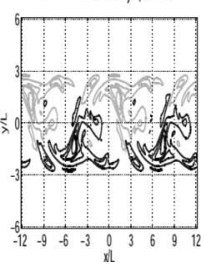

FIG. 22. Nonlinear evolution of the most unstable mode (Ro = 1.5) of Fig. 14 (see Fig. 15). 1st (2nd) row: Isolines of the relative vorticity of the upper (lower) layer. Contour interval $0.5 R o \beta L$, starting at the value \pm 0.5 Ro $\beta L$ (positive/negative relative vorticity anomaly: black/gray lines).
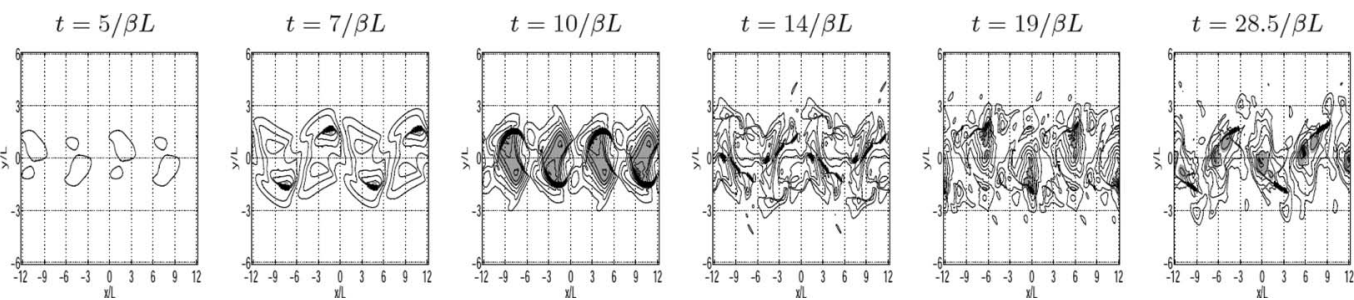

FIG. 23. Nonlinear evolution of the most unstable mode $(R o=1.5)$ of Fig. 14 (see Fig. 15). Isolines of the velocity shear $\mid u_{2}$ $-u_{1} \mid$ (thin black lines), enhanced dissipation zones (black), and zones of hyperbolicity loss (gray). Contour interval $0.25 U_{0}$ for $\left|u_{2}-u_{1}\right|$.

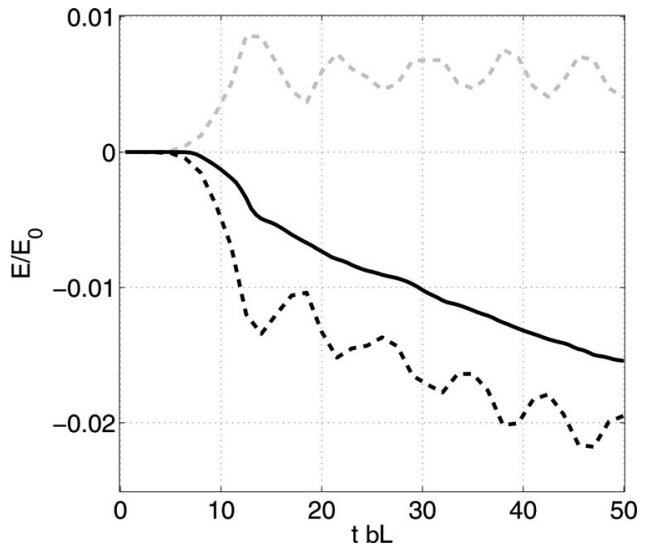

FIG. 24. Nonlinear evolution of the most unstable mode $(R o=1.5)$ of Fig. 14 (see Fig. 15). Normalized deviation of the total (black), kinetic (black dashed), and potential (gray dashed) energy in the whole calculation domain from their initial values.

to the loss of hyperbolicity are confirmed by Figs. 23 and 24. The events of hyperbolicity loss (and hence Kelvin-Helmholtz instability) persist until very late stages of the evolution (not shown).

The above-described mixing leads to the reorganization of the flow. We give in Fig. 25, a comparison of the initial velocity profile of the jet with that of the late stages of the evolution. As discussed in Sec. IV B 3, inertial instability is active at the considered value of $R o$, which means 

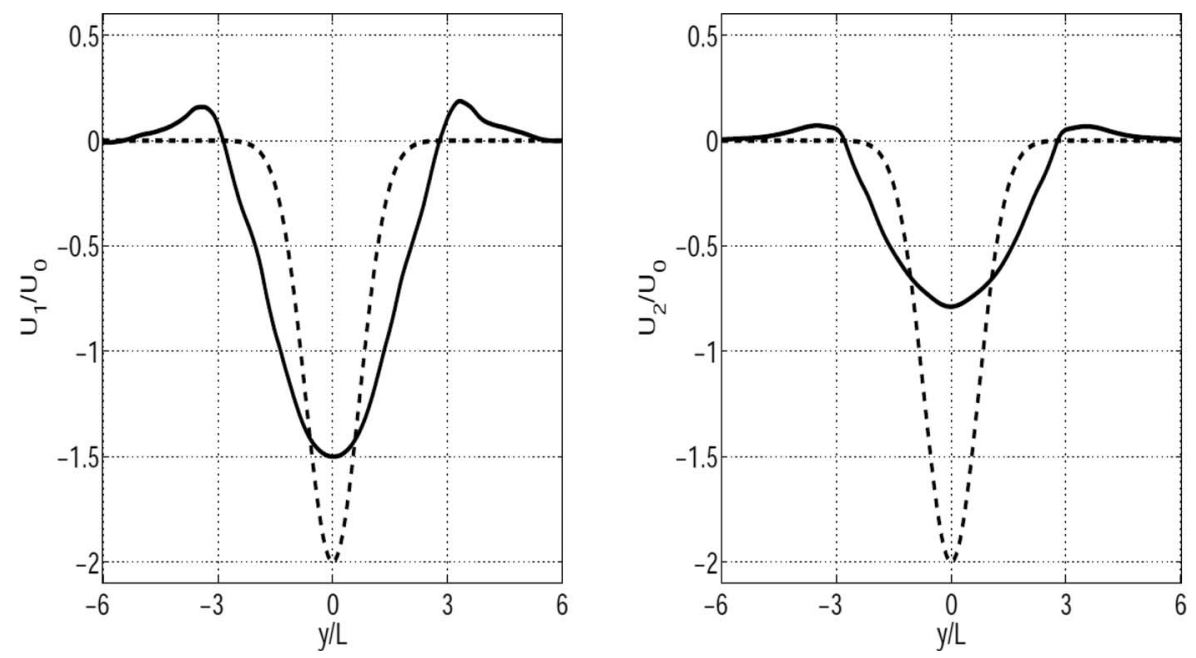

FIG. 25. Initial (dashed) vs late $(t=31.5 / \beta L$, solid) mean velocity profile of the jet. (Left) - upper layer; (Right) - lower layer.

that the product of planetary and potential vorticities $\beta y \cdot q_{i}, i=1,2$ is negative somewhere in the flow. This is, indeed, the case, but the above-described reorganization of the flow "cures" the inertial instability rendering these quantities everywhere non-negative in each layer, as shown in Fig. 26. This is typical for the saturation of inertial instability. ${ }^{35,18}$

\section{A résumé of the saturation}

Our results show that in the one-layer configuration the dominant in the ageostrophic regime YY instability saturates by forming a vortex street which experiences periodic oscillations. In the two-layer configuration, the barotropic and baroclinic YY instabilities saturate differently. While the former, roughly, follows the same scenario as its one-layer counterpart, the latter produces a lot of mixing and small-scale structures, especially in the upper layer.
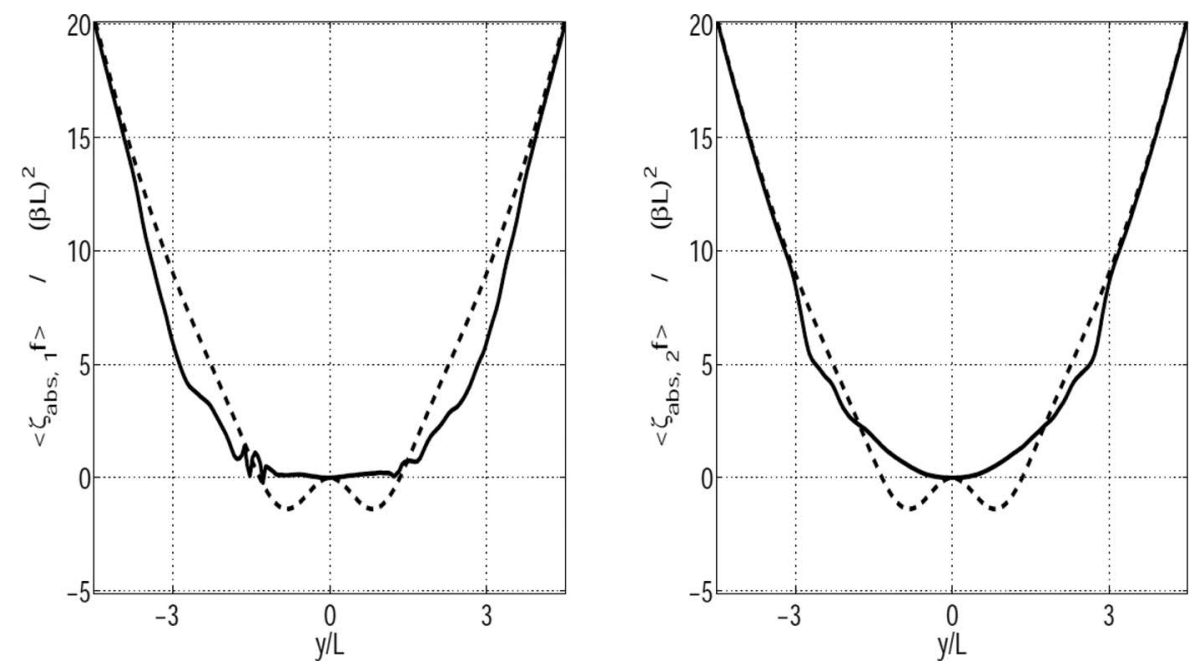

FIG. 26. Initial (dashed) vs late $\left(t=31.5 / \beta L\right.$, solid) profile of $\beta y \cdot \zeta_{\text {abs }}$ normalized by $(\beta L)^{2}$. (Left) - upper layer; (Right) - lower layer. 


\section{SUMMARY AND DISCUSSION}

We have analyzed the linear stability of the Gaussian easterly jet in one- and two-layer versions of the shallow-water model on the equatorial beta-plane and shown that dominant instability in the long-wave sector is due to the phase-locking and resonance of the Yanai waves propagating on the background of the jet (YY instability). In the two-layer case, this instability may be either barotropic or baroclinic, the baroclinic being stronger, at large Rossby numbers.

We have shown that at fixed ageostrophic (of the order one) value of the Rossby number and Burger number diminishing towards its limiting in the model values (determined by in- or outcropping threshold), the distribution of the growth rates of the YY-instability shifts towards zero in the wavenumber space, and eventually develops a non-zero limit at zero along-jet wavenumbers, which corresponds to the classical symmetric inertial instability. Yet, the maximal growth rate corresponds to small but non-zero $k$ (asymmetric inertial instability). This instability may be barotropic (necessarily in the one-layer version, but arising in the two-layer one, too) or baroclinic, the last one being always stronger. At low enough Burger numbers higher meridional modes of symmetric instability appear. With increasing Rossby number at fixed large Burger number the baroclinic YY-instability in the two-layer case also shifts towards smaller wavenumbers $k$ and finally reaches $k=0$ with nonzero growth rate.

This behavior contrasts the situation on the mid-latitude $f$-plane, where symmetric barotropic inertial instability is impossible in the one-layer model. In the two-layer model, the behavior is similar to what was observed for baroclinic instability on the $f$-plane in Ref. 6 . It should be mentioned that ${ }^{23}$ demonstrated that in the continuously stratified flows the growth rate of the inertial instability has a (local) maximum at $k=0$, i.e., for the symmetric instability, in the limit of infinite vertical wavenumbers. Yet the results of Ref. 36 and the recent ones by Ref. 8 on the mid-latitude tangent plane show that there is a wide range of vertical wavenumbers where the asymmetric inertial instability is stronger. We, thus, expect the present result on the dominance of asymmetric instability to be valid for a range of vertical wavenumbers of the perturbations of the equatorial jets in the continuously stratified case. In this context, we should emphasize that we do see a tendency in the growth-rate curves to form a local extremum at $k=0$ which is, however, always accompanied by a global maximum at small but non-zero $k$.

Nonlinear saturation of the dominant instability displays formation of the vortex street subject to nonlinear oscillations in the barotropic case, and generation of strong vertical shears and related dissipation and mixing in the baroclinic case. It is worth emphasizing that, with respect to the similar analysis on the mid-latitude $f$-plane,${ }^{6}$ we do not observe the formation of intense secondary vortices, and the meridional spreading of the flow is lesser, which is consistent with the beta-plane "rail" character. This means that in order to produce intense secondary detaching vortices, the instability of the easterly jets per se is insufficient, and some extra effects are needed, such as convective forcing and/or topography. Both may be easily included in the numerical scheme, and their effects will be studied elsewhere.

\section{ACKNOWLEDGMENTS}

This work was supported by the French ANR grant "SVEMO." V.Z. is grateful to P. Read for a fruitful discussion and useful references. We are grateful to the anonymous reviewers for critical reading of the paper and useful suggestions and references.

\section{APPENDIX A: INTEGRAL ESTIMATES OF THE EIGENFREQUENCIES OF THE TRAPPED MODES IN THE ONE-LAYER MODEL UNDER CONSTRAINT OF STRICT ZONAL SYMMETRY}

Equations (2.1) in the strictly zonally symmetric case, when all derivatives with respect to the meridional coordinate vanish, take the following form: 


$$
\begin{aligned}
u_{t}+v u_{y}-\beta y v & =0, \\
v_{t}+v v y+\beta_{y} u & =-g h_{y}, \\
h_{t}+(h v)_{y} & =0,
\end{aligned}
$$

where the subscripts denote the corresponding partial derivatives. By linearizing around the background state (2.4), eliminating $u$ and $h$ in favor of $v$, and making the Fourier-transform in $t$ one arrives to the following equation for $v(y)$ :

$$
-\omega^{2} v+\beta y\left(\beta y-U^{\prime}(y)\right) v-g H(y) v^{\prime \prime}=0,
$$

where the prime denotes the derivative with respect to $y$. An integral estimate for $\omega^{2}$ of trapped (i.e., fast decaying in $y$ ) modes easily follows:

$$
\omega^{2}=\frac{\int_{-\infty}^{+\infty} d y \frac{\beta y\left(\beta y-U^{\prime}\right)}{g H}|v|^{2}+\int_{-\infty}^{+\infty} d y\left|v^{\prime}\right|^{2}}{\int_{-\infty}^{+\infty} d y \frac{1}{g H}|v|^{2}} .
$$

One may infer from this estimate that for westward intense enough jets the first term in the numerator may became negative and overcome the second term, while the denominator is positive-definite, thus giving (symmetric) instability. Moreover, if non-dimensionalized as in the main text, this expression shows that the first term in the numerator contains the combination $R o-1 / 4$ related to the Ripa's criterion - see Appendix B below - and that the second term is proportional to $B u$, thus indicating that instability is facilitated by low values of $B u$.

Nevertheless, this estimate may be misleading because following the same lines on the $f$-plane with a jet in the geostrophic equilibrium with the thickness profile $H(y)$, one gets

$$
\omega^{2}=f^{2}+\frac{\int_{-\infty}^{+\infty} d y \frac{H^{\prime \prime}}{H}|v|^{2}+\int_{-\infty}^{+\infty} d y\left|v^{\prime}\right|^{2}}{\int_{-\infty}^{+\infty} d y \frac{1}{g H}|v|^{2}},
$$

and may also infer an instability, as the first term in the numerator is not positive-definite. Yet, as is was shown in Ref. 26 by using Lagrangian approach in order to get another integral estimate, the jets on the $f$-plane are not only symmetrically stable, but all frequencies of zonally symmetric trapped eigenmodes are supra-inertial. By repeating the approach of Ref. 26 on the equatorial $\beta$-plane we get the following equations for the Lagrangian coordinate $Y(y, t)$ :

$$
\begin{aligned}
\partial_{t}^{2} Y+\beta Y u+g \partial_{Y} h & =0, \\
\partial_{t} u-\beta y \partial_{t} Y & =0, \\
h-h_{I}(y) \frac{1}{\partial_{y} Y} & =0,
\end{aligned}
$$

where $h_{I}$ is the initial thickness distribution. By linearizing around the geostrophically balanced zonal jet with velocity $u_{I}\left(u_{I}, h_{I}\right.$ replace $U, H$ above), and introducing the departures of Lagrangian particles from their initial positions $Y(y, t)=y+\xi(y, t)$, we get the following equation for $\xi$ :

$$
\partial_{t t} \xi+\left(u_{I}+\beta y^{2}\right) \beta \xi-g h_{I} \partial_{y y} \xi-2 g h_{I}^{\prime} \partial_{y} \xi=0
$$

whence, after Fourier-transforming $\xi$ in time $\xi=\tilde{\xi} e^{i \omega t}$, we get the integral estimate for the eigenfrequency squared

$$
\omega^{2}=\frac{\int_{-\infty}^{+\infty} d y \beta\left(\beta y^{2}+u_{I}\right) g h_{I}|\tilde{\xi}|^{2}+\int_{-\infty}^{+\infty} d y g^{2} h_{I}^{2}\left|\tilde{\xi}^{\prime}\right|^{2}}{\int_{-\infty}^{+\infty} d y g h_{I}|\tilde{\xi}|^{2}} .
$$

Unlike its $f$-plane conterpart

$$
\omega^{2}=f^{2}+\frac{\int_{-\infty}^{+\infty} d y g^{2} h_{I}^{2}\left|\tilde{\xi}^{\prime}\right|^{2}}{\int_{-\infty}^{+\infty} d y g h_{I}|\tilde{\xi}|^{2}}
$$

the Lagrangian estimate (A7) does not prevent symmetric instability for strong enough westward flows, which constitutes an essential difference between the $f$-plane and the equatorial $\beta$-plane. 


\section{APPENDIX B: A CHECK OF RIPA'S STABILITY CRITERION IN THE ONE-LAYER MODEL}

In this Appendix, the Ripa's general stability conditions are checked in the one-layer configuration. The two-layer configuration can be easily treated along the same lines.

In order to avoid outcropping, the aspect ratio $\lambda=\frac{\Delta H}{H_{0}}$ is bounded by $|\lambda|=\frac{R o}{B u}<1$, where $R o$ and $B u$ are, respectively, the Rossby and the Burger numbers. We are a priori interested in high values of the Rossby number, and thus Burger number cannot be very small. We thus assume that $B u>1 / 4$. In addition, $\Delta H$ is considered to be positive, so that the vorticity $\zeta=-\partial_{y} U$ times the Coriolis parameter $\beta y$ is negative.

The non-dimensionalized layer depth $H$, velocity $U$, and potential vorticity $Q$ are denoted by an overbar below, as well as non-dimensional meridional coordinate $\bar{y}=y / L$. We also introduce a shorthand notation $\varphi(\bar{y})=e^{-\bar{y}^{2}}$. The background flow then becomes

$$
\begin{aligned}
& \bar{H}(\bar{y})=1-\lambda \varphi(\bar{y}), \\
& \bar{U}(\bar{y})=-2 \varphi(\bar{y}), \\
& \bar{Q}(\bar{y})=\bar{y} \frac{1-4 \operatorname{Ro} \varphi(\bar{y})}{1-\lambda \varphi(\bar{y})} .
\end{aligned}
$$

In order to check the Ripa's general stability criteria, we need to determine the sign of meridional gradient of $\mathrm{PV} \frac{\mathrm{d} \bar{Q}}{\mathrm{~d} \bar{y}}$. A straightforward calculation gives

$$
\begin{aligned}
\frac{\mathrm{d} \bar{Q}}{\mathrm{~d} \bar{y}}(1-\lambda \varphi(\bar{y}))^{2} & =1+4 \operatorname{Ro} \lambda \varphi^{2}(\bar{y})-\left(4 B u+1-2(4 B u-1) \bar{y}^{2}\right) \lambda \varphi(\bar{y}) \\
& \equiv \mathfrak{F}(\bar{y}), \\
\frac{\mathrm{d} \mathfrak{F}(\bar{y})}{\mathrm{d} \bar{y}} & =2 \bar{y} \varphi(\bar{y})\left(8 \operatorname{Ro} \lambda(1-\varphi(\bar{y}))+8 \operatorname{Ro}(1-\lambda)-\lambda(4 B u-1)\left(2 \bar{y}^{2}-1\right)\right) \\
& \equiv 2 \bar{y} \varphi(\bar{y}) \mathfrak{G}(\bar{y}) .
\end{aligned}
$$

If $4 B u>1$, there exists a unique $\overline{y_{0}}>0$, such that $\mathfrak{G}\left(|\bar{y}|<\overline{y_{0}}\right)>0$ and $\mathfrak{G}\left(|\bar{y}|>\overline{y_{0}}\right)<0$. Thus the sign of the slope of the function $\mathfrak{F}$ is known. $\mathfrak{F}$ is an even function that increases in the interval $\left[0 ; \overline{y_{0}}\right]$ and decreases in the interval $\left[\overline{y_{0}} ;+\infty[\right.$. This function has limits $\mathfrak{F}(\bar{y}= \pm \infty)=1$ and a local minimum in $\mathfrak{F}(\bar{y}=0)=(1-4 R o)(1-\lambda) .4 R o<1$ implies thus that the meridional gradient $\frac{\mathrm{d} \bar{Q}}{\mathrm{~d} \bar{y}}$ of the potential vorticity is always positive. Hence, for all $\alpha>0$ the function $(\bar{U}-\alpha) \frac{\mathrm{d} \bar{Q}}{\mathrm{~d} \bar{y}}$ remains negative. On the contrary, $4 R o>1$ implies that $\mathfrak{F}(\bar{y}=0)<0$. Therefore, the meridional gradient $\frac{\mathrm{d} \bar{Q}}{\mathrm{~d} \bar{y}}$ of the potential vorticity is negative near $\bar{y}=0$, and positive elsewhere, and there is thus no such $\alpha$ for which $(\bar{U}-\alpha) \frac{\mathrm{d} \bar{Q}}{\mathrm{~d} \bar{y}}$ remains always negative. Therefore, the flow satisfies the first criterion of Ripa's general stability conditions only if $4 R o \leq 1$.

Once non-dimensionalized, the 2 nd criterion is

$$
\begin{gathered}
\bar{H}(\bar{y}) \geq \lambda \operatorname{Ro}(\bar{U}(\bar{y})-\alpha)^{2}, \\
\Longleftrightarrow \quad 1-\lambda \varphi(\bar{y}) \geq \lambda \operatorname{Ro}(2 \varphi(\bar{y})+\alpha)^{2}, \\
\Longleftrightarrow \varphi(\bar{y})^{2}+\left(\alpha+\frac{1}{4 \operatorname{Ro}}\right) \varphi(\bar{y})+\frac{1}{4}\left(\alpha^{2}-\frac{1}{\lambda \operatorname{Ro}}\right) \leq 0 .
\end{gathered}
$$

For $\alpha^{2} \lambda R o \leq 1$, the second criterion of Ripa's general stability conditions is satisfied if $\frac{1}{4 R o} \geq 1$. In summary, the flow is stable if $4 R o<1$. 
${ }^{1}$ S. G. H. Philander, "Instabilities of zonal equatorial currents," J. Geophys. Res. 81, 3725-3735, doi:10.1029/JC081i021p03725 (1976).

${ }^{2}$ P. Chatterjee and B. N. Goswami, "Structure, genesis and scale selection of the tropical quasi-biweekly mode," Q. J. R. Met. Soc. 130, 1171 (2004).

3 J. L. Mitchell and G. K. Vallis, "The transition to superrotation in terrestrial atmospheres," J. Geophys. Res. 115, E12008, doi:10.1029/2010JE003587 (2010).

${ }^{4}$ B. L. Hua, D. W. Moore, and S. Le Gentil, "Inertial nonlinear equilibration of equatorial flows," J. Fluid Mech. 331, 345-371 (1997).

${ }^{5}$ S. D. Griffiths, "The nonlinear evolution of zonally symmetric equatorial inertial instability," J. Fluid Mech. 474, 245-273 (2003).

${ }^{6} \mathrm{~F}$. Bouchut, B. Ribstein, and V. Zeitlin, "Inertial, barotropic, and baroclinic instabilities of the Bickley jet in two-layer rotating shallow water model," Phys. Fluids 23, 126601 (2011).

${ }^{7}$ G. F. Carnevale, R. C. Kloosterziel, and P. Orlandi, "Inertial and barotropic instabilities of a free current in three-dimensional rotating flow," J. Fluid Mech. 725, 117-151 (2013).

${ }^{8}$ B. Ribstein, R. Plougonven, and V. Zeitlin, "Inertial vs baroclinic instability of the Bickley jet in continuously stratified fluid," J. Fluid Mech. 743, 1-31 (2014).

${ }^{9}$ J. P. Holton, An Introduction to Dynamic Meteorology (Academic Press, NY, 1992).

${ }^{10}$ A. Gill, "Some simple solutions for heat-induced tropical circulation," Q. J. R. Met. Soc. 106, 447-462 (1980).

${ }^{11}$ D. A. Schecter and T. J. Dunkerton, "Hurricane formation in diabatic Ekman turbulence," Q. J. R. Met. Soc. 135, 823-838 (2009).

12 A. Gill, Atmosphere-Ocean Dynamics (Academic Press, London, 1982).

${ }^{13}$ F. Bouchut and V. Zeitlin, "A robust well-balanced scheme for multi-layer shallow water equations,” Disc. Cont. Dyn. Syst. B 13, 739-758 (2010).

${ }^{14}$ Y.-Y. Hayashi and W. R. Young, "Stable and unstable shear modes of rotating parallel flows in shallow water," J. Fluid Mech. 184, 477-504 (1987).

${ }^{15}$ M. Renardy and J. Swaters, "Stability of equatorial currents with nonzero potential vorticity," Geophys. Astrophys. Fluid Dyn. 85, 31-64 (1997).

${ }^{16} \mathrm{H}$. Taniguchi and M. Ishiwatari, "Physical interpretation of unstable modes of a linear shear flow in shallow water on an equatorial beta-plane," J. Fluid Mech. 567, 1-26 (2006).

${ }^{17}$ S.-I. Iga and Y. Matsuda, "Shear instability in a shallow water model with implications for the venus atmosphere," J. Atmos. Sci. 62, 2514-2527 (2005)

${ }^{18}$ R. C. Kloosterziel, P. Orlandi, and G. F. Carnevale, "Saturation of equatorial inertial instability," personal communication (2014).

${ }^{19}$ V. Zeitlin, in Nonlinear Dynamics of Rotating Shallow Water: Methods and Advances, edited by V. Zeitlin (Elsevier, Amesterdam, 2007)

${ }^{20} \mathrm{M}$. Wheeler and G. N. Kiladis, "Convectively coupled equatorial waves: Analysis of clouds and temperature in the wavenumber-frequency domain,” J. Atmos. Sci. 56, 374-399 (1999).

${ }^{21}$ J. Le Sommer, H. Teitelbaum, and V. Zeitlin, "Global estimates of equatorial inertia-gravity wave activity in the stratosphere inferred from ERA40 reanalysis," Geophys. Res. Lett. 33, L07810, doi:10.1029/2005GL024873 (2006).

${ }^{22}$ J.-I. Yano and M. Bonazzola, "Scale analysis for large-scale tropical atmospheric dynamics," J. Atmos. Sci. 66, 159-172 (2009).

${ }^{23}$ S. D. Griffiths, "The limiting form of inertial instability in geophysical flows," J. Fluid Mech. 605, 115-143 (2008).

${ }^{24}$ P. Ripa, "General stability conditions for a multi-layer model," J. Fluid Mech. 222, 119-137 (1991).

${ }^{25}$ L. N. Trefethen, Spectral Methods in Matlab (SIAM, Philadelphia, 2000).

${ }^{26}$ V. Zeitlin, S. B. Medvedev, and R. Plougonven, "Frontal geostrophic adjustment, slow manifold and nonlinear wave phenomena in one-dimensional rotating shallow water. Part 1. Theory," J. Fluid Mech. 481, 269-290 (2003).

${ }^{27}$ R. Plougonven and V. Zeitlin, "Nonlinear development of inertial instability in a barotropic shear," Phys. Fluids 21, 106601 (2009).

${ }^{28}$ F. Bouchut, J. Le Sommer, and V. Zeitlin, "Breaking of equatorial waves," Chaos 15, 013503 (2005).

${ }^{29}$ F. L. Poulin and G. R. Flierl, "The nonlinear evolution of barotropically unstable jets," J. Phys. Oceanogr. 33, 2173-2192 (2003).

${ }^{30}$ J. Lambaerts, G. Lapeyre, and V. Zeitlin, "Moist vs dry barotropic instability in a shallow water model of the atmosphere with moist convection," J. Atmos. Sci. 68, 1234-1252 (2011).

${ }^{31}$ S. Sakai, "Rossby-Kelvin instability: A new type of ageostrophic instability caused by a resonance between Rossby waves and gravity waves," J. Fluid Mech. 202, 149-176 (1989).

${ }^{32}$ T. J. Dunkerton, “A nonsymmetric equatorial inertial instability,” J. Atmos. Sci. 38, 807-813 (1982).

${ }^{33}$ F. Bouchut, in Nonlinear Dynamics of Rotating Shallow Water: Methods and Advances, edited by V. Zeitlin (Elsevier, Amesterdam, 2007).

${ }^{34}$ J. Le Sommer, S. B. Medvedev, R. Plougonven, and V. Zeitlin, "Singularity formation during relaxation of jets and fronts toward the state of geostrophic equilibrium," Commun. Nonlin. Sci. Num. Simul. 8, 415-442 (2003).

${ }^{35}$ R. C. Kloosterziel, P. Orlandi, and G. F. Carnevale, "Saturation of inertial instability in rotating planar shear flows," J. Fluid Mech. 583, 413-422 (2007)

${ }^{36}$ D. E. Stevens and P. E. Ciesielski, "Inertial instability of horizontally sheared flow away from the equator," J. Atmos. Sci. 43, 2845-2856 (1986). 